231640

UCRL-ID-127109

\title{
Computational Model of a Copper Laser
}

\author{
C. D. Boley
}

W. A. Molander

B. E. Warner

March 26, 1997

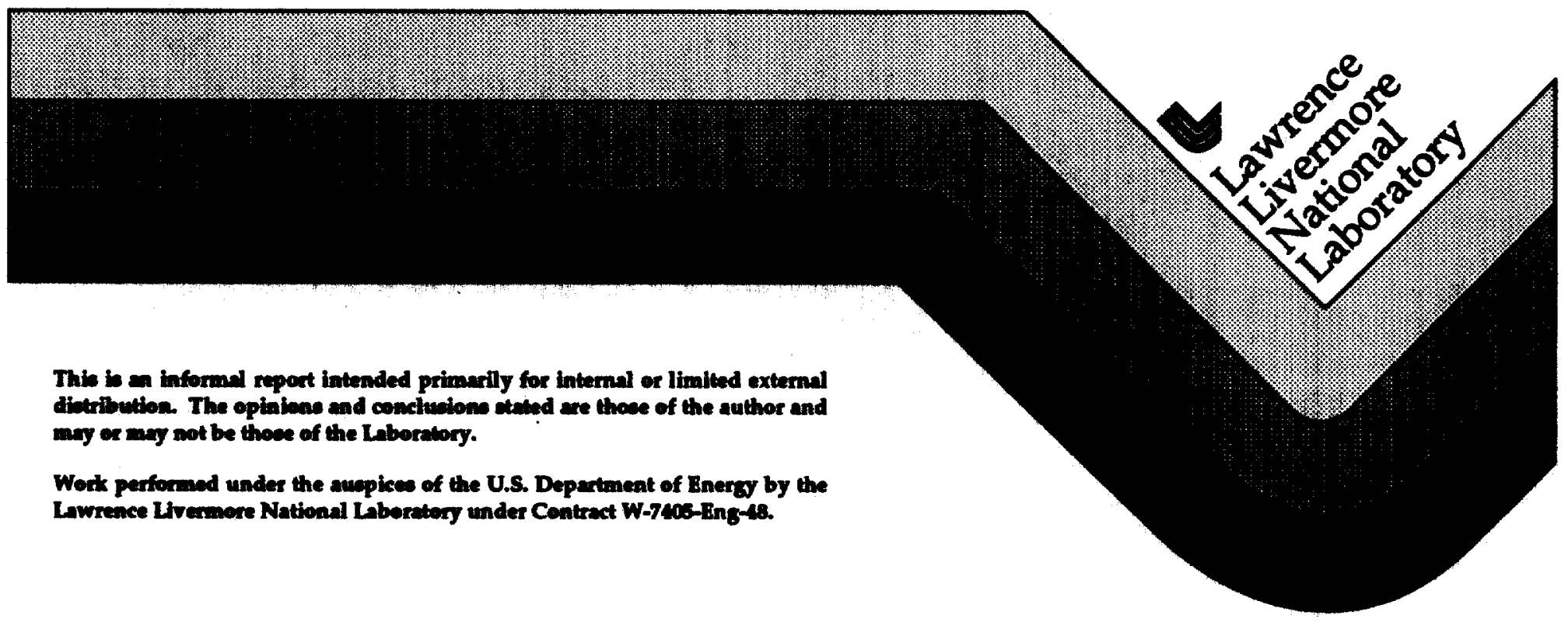




\section{DWCLAMIER}

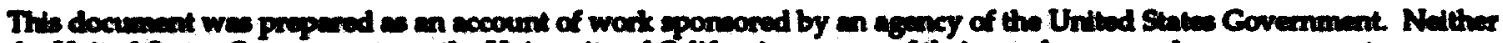

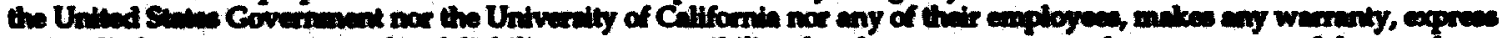

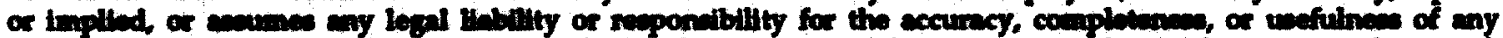

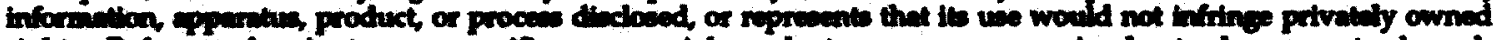

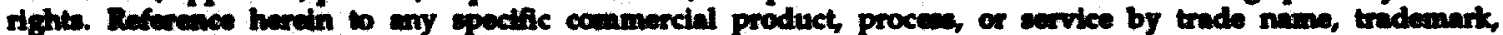

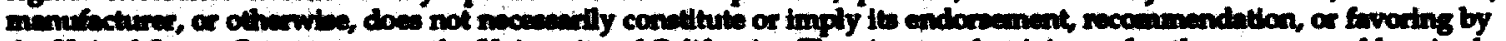

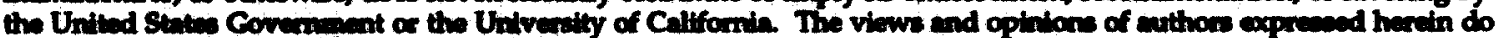

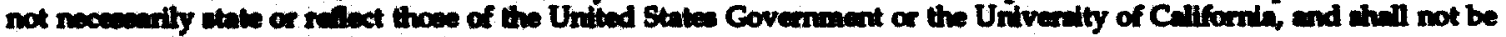
used for adverting or product endonsment purposea.

This report hes been reproduced directly from the beot aviliable copy.

Aveluble to DOE and DOB contructors from the Orice of Solmitific and Techricel hiformation P.O. Box 62, Oak RLdge, TN 37331

Prices avalibble from (615) 5\%6-401, FIs 626-8401

Avellable to the priblic from the

Nationel Technical information Service

U.S. Department of Commerce

5285 Port Royal Rd.

Sprtingfield VA 22161 


\title{
Computational Model of a Copper Laser
}

\author{
C. D. Boley, W. A. Molander, and B. E. Warner \\ University of California \\ Lawrence Livermore National Laboratory \\ Livermore, CA 94550
}

\begin{abstract}
This report describes a computational model of a copper laser amplifier. The model contains rate equations for copper and the buffer gas species (neon and hydrogen), along with equations for the electron temperature, the laser intensity, and the diffusing magnetic field of the discharge. Rates are given for all pertinent atomic reactions. The radial profile of the gas temperature is determined by the time-averaged power deposited in the gas. The presence of septum inserts, which aid gas cooling, is taken into account. Fields are calculated consistently throughout the plasma and the surronnding insulation. Employed in conjunction with a modulator model, the model is used to calculate comprehensive performance predictions for a high-power operational amplifier.
\end{abstract}

\section{Introduction}

The copper laser is characterized by laser transitions from an excited pair of states to a low-lying metastable pair [1]. The lasing transitions, of wavelengths $510.6 \mathrm{~nm}$ and $578.2 \mathrm{~nm}$, are terminated after some 50-70 ns by accumulation of atoms in the lower states. The laser is important not only because of its visible wavelengths and short pulse length, but also because of its high repetition rate (several kilohertz), its 1-2\% electrical efficiency, and the practicality of nearly diffraction-limited operation at an average power of hundreds of watts $[2,3]$. During the last two decades, copper lasers have been developed extensively at Lawrence Livermore National Laboratory [2-10], as well as in Russia and the former USSR [11-40], Israel [41-47], Japan [48-56], the UK [57-60], Australia [61-68], and elsewhere.

The subject of this report is a computational model of a copper laser amplifier, as constituted in a computer code. The essence of the model is a set of rate equations for the states of the constituent species (copper, neon, and hydrogen), the electron temperature, the extracted laser intensity, and the magnetic field of the discharge. These equations are coupled to a modulator model [69] by the boundary value of the magnetic field, which gives the plasma current.

In our model, there are seven copper species, three neon species, and five species of atomic and molecular hydrogen. The processes described in the rate equations include electronimpact excitation, deexcitation, and ionization, line radiation with trapping effects, optical pumping, three-body recombination, charge exchange, Penning ionization, and (in the case of hydrogen) molecular dissociation, recombination, and negative-ion attachment/detachment. 
The electron temperature equation includes ohmic heating, cooling via inelastic collisions, and equilibration with the gas temperature via elastic collisions, along with effects from other reactions. Detailed rates are given for all reactions.

Another quantity in the model is the gas temperature (the common temperature of atoms and ions), which rises markedly at the center of the discharge and is held to the wall temperature at the boundaries. In view of the large thermal inertia of the system, the gas temperature changes little during a single pulse. It is calculated via a steady state model, in which the radial profile is determined by the time-averaged power deposited in the gas. Recently it has been shown [9] that septa placed vertically along the length of the tube are effective in lowering the gas temperature. This benefits the discharge both by decreasing the prepulse electron temperature and by raising the gas density and thus the discharge impedance. The effects of septa are included in the model.

The model contains a detailed description of field diffusion across the discharge, and it provides the fields within the insulation package. In the version of the model discussed here, the various species are assumed to have a radial profile corresponding to the reciprocal of the gas temperature. This is expected to provide a reasonable approximation to the radial distribution. A version including radial particle and energy diffusion has been developed in large part, but circumstances have impeded its completion.

Several computational models of a copper laser have been developed during the last decades $[4,6,7,16,21,25,70,71,72]$, of which the model of Kushner and Warner [7] is closest in spirit to ours. Relative to these, our model has greatly improved treatments of reaction rates, gas heating, and electromagnetics. A recent model by Carman et al. [65] has been shown to predict successfully the dynamics of a small-bore laser. Its treatment of the neutral copper atom appears roughly equivalent to ours, although we believe that our rates are more current. It includes two elevated CuII levels, which we neglect. It contains ambipolar and neutral diffusion as well as electron thermal transport, but neglects field diffusion. A two-temperature treatment of the electron energy distribution function is constructed. The geometry is that of a cylindrical tube without septa.

In Section 2, we describe the atomic physics models of copper, neon, and hydrogen. In Section 3, we discuss the discharge dynamics and, in particular, the rate equations for the various species and the electron temperature. Then we turn to the gas heating model (Section 4) and the plasma electromagnetics (Section 5). The computational scheme is outlined in Section 6. In Section 7 we turn specifically to a septum laser and present results describing both a representative operating point and a systematic survey of operating points. Our conclusions are given in Section 8. Four appendices contain details of the atomic rates and the treatment of radiation trapping.

\section{Atomic States of the Model}

\subsection{Copper States}

In the copper atom, the lasing transitions begin on $J$ multiplets of the $(4 \mathrm{p})^{2} \mathrm{P}$ configuration and end on $\mathrm{J}$ multiplets of the $\left(4 \mathrm{~s}^{2}\right)^{2} \mathrm{D}$ configuration. These are also the configurations lying 
just above the ground state. The next several higher neutral states play a role in depopulating the upper laser level via electron impact collisions, but that role is expected to diminish for larger excitation energies. The ionized population, however, always is significant, in view of the slow rate of recombination. A reasonable model of the copper atom would therefore contain the ground state, the lasing states (each divided into two J multiplets), an aggregate of the next several states, and the first ionized state. Thus our $\mathrm{Cu}$ model has the seven effective states listed in Table 1. The energies are taken from the tabulation by Moore [73]. Here and elsewhere, the approximate electron configurations are abbreviated according to the terminology of Table 2 .

Table 1: Cu States of the Model
\begin{tabular}{|lc|}
\hline State & $\mathrm{E}(\mathrm{eV})$ \\
\hline$(1)(4 \mathrm{~s})^{2} \mathrm{~S}_{1 / 2}$ & 0 \\
$(2)\left(4 \mathrm{~s}^{2}\right)^{2} \mathrm{D}_{5 / 2}$ & 1.389 \\
$(3)\left(4 \mathrm{~s}^{2}\right)^{2} \mathrm{D}_{3 / 2}$ & 1.642 \\
$(4)(4 \mathrm{p})^{2} \mathrm{P}_{1 / 2}^{\circ}$ & 3.786 \\
$(5)(4 \mathrm{p})^{2} \mathrm{P}_{3 / 2}^{\circ}$ & 3.816 \\
(6) $\mathrm{Pseudostate}^{\circ}$ & - \\
(7) $\mathrm{Cu}^{+}$ & 7.724 \\
\hline
\end{tabular}

Table 2: Abbreviations for Electron Configurations

\begin{tabular}{|ll|}
\hline $3 d^{10} 4 s$ & $(4 s)$ \\
$3 d^{9} 4 s^{2}$ & $\left(4 s^{2}\right)$ \\
$3 d^{10} 4 p$ & $(4 p)$ \\
$3 d^{9} 4 s 4 p$ & $\left(4 p^{\prime}\right)$ \\
$3 d^{10} 5 s$ & $(5 s)$ \\
$3 d^{10} 5 p$ & $(5 p)$ \\
$3 d^{10} 4 d$ & $(4 d)$ \\
\hline
\end{tabular}

In the notation of Table 1, the green lasing transition $(510.6 \mathrm{~nm})$ connects states 5 and 2 , while the yellow transition $(578.2 \mathrm{~nm})$ connects states 4 and 3 .

The aggregate state, or pseudostate, represents an average of the nine states immediately above $(4 \mathrm{p})^{2} \mathrm{P}_{3 / 2}^{o}$. The configurations and energies of these states are listed in Table 3 . We assume that the relative populations of these states are distributed according to local thermodynamic equilibrium at electron temperature $T_{e}$. The effective degeneracy is given by

$$
g_{p}\left(T_{e}\right)=\sum_{i=1}^{9} g_{i} \exp \left[-\left(E_{i}-E_{1}\right) / T_{e}\right],
$$


Table 3: Cu States Represented by Pseudostate

\begin{tabular}{|lll|}
\hline & State & $\mathrm{E}(\mathrm{eV})$ \\
\hline$(1)$ & $\left(4 \mathrm{p}^{\prime}\right)^{4} \mathrm{p}^{\circ}$ & 4.922 \\
$(2)$ & $\left(4 \mathrm{p}^{\prime}\right)^{4} \mathrm{~F}^{\circ}$ & 5.122 \\
$(3)$ & $(5 \mathrm{~s})^{2} \mathrm{~S}$ & 5.348 \\
$(4)$ & $\left(4 \mathrm{p}^{\prime}\right)^{4} \mathrm{D}^{\circ}$ & 5.471 \\
$(5)$ & $\left(4 \mathrm{p}^{\prime}\right)^{2} \mathrm{~F}^{\circ}$ & 5.508 \\
$(6)$ & $\left(4 \mathrm{p}^{\prime}\right)^{2} \mathrm{p}^{\circ}$ & 5.685 \\
$(7)$ & $\left(4 \mathrm{p}^{\prime}\right)^{2} \mathrm{D}^{\circ}$ & 5.756 \\
$(8)$ & $(5 \mathrm{p})^{2} \mathrm{P}^{\circ}$ & 6.122 \\
$(9)$ & $(4 \mathrm{~d})^{2} \mathrm{D}$ & 6.191 \\
\hline
\end{tabular}

where the sum runs over the levels of Table 3. At low $T_{e}$, this equals the degeneracy of $\left(4 \mathrm{p}^{\prime}\right)^{4} \mathrm{P}^{\circ}$, while at temperatures near $10 \mathrm{eV}$ it approaches the sum of the pseudostate degeneracies, $g_{p} \rightarrow 108$, as shown in Fig. 1 . The effective energy of the pseudostate, also shown in Fig. 1, increases from about $4.92 \mathrm{eV}$ [i.e. the energy of $\left(4 \mathrm{p}^{\prime}\right)^{4} \mathrm{P}^{\circ}$ ] at $T_{e}=0.1 \mathrm{eV}$ to about $5.45 \mathrm{eV}$ at $T_{e}=10 \mathrm{eV}$.

Given these copper states, we require radiation rates, excitation/deexcitation rates, ionization rates, and momentum transfer rates. All of these are discussed in detail in Appendix A.

\subsection{Neon States}

Since the first excited state of the neon atom has an energy of more than $16 \mathrm{eV}$, our representation of the Ne atom is quite simple. We use a three-state model consisting of the ground state, a pseudostate representing the $3 \mathrm{~s}$ and $3 p$ levels, and the first ionized state. The pseudostate is constructed as a statistical average, in the same spirit as the $\mathrm{Cu}$ pseudostate, with energy levels taken from Moore [74].

The principal role of neon is to increase the laser impedance. Hence electron-atom momentum transfer is the process of primary importance. The rates for this and the other pertinent processes are given in Appendix B.

\subsection{Hydrogen States}

The addition of about $0.5 \%$ hydrogen is known to increase the laser efficiency [ 75,76$]$. In our model, the main effect of hydrogen is to enhance the electron-atom energy exchange rate. That rate varies directly with the momentum transfer rate and inversely with the atomic mass. The momentum transfer cross section of atomic hydrogen is the same order of magnitude as that of neon, and that of molecular hydrogen is nearly an order of magnitude larger. The masses of $\mathrm{H}$ and $\mathrm{H}_{2}$ contribute factors of 20 and 10, respectively, to the energy exchange rate. Thus the quiescent electron temperature is lowered, decreasing the prepulse 
metastable copper populations and increasing the optical power. This beneficial effect ceases at a concentration (on the order of a percent) at which the hydrogen overionizes.

Our model includes the hydrogen ground state, an aggregate excited state composed of the $n=2$ and $n=3$ levels, the hydrogen ion, the negative ion, and the ground state of the hydrogen molecule. In practice, only the ground state populations are significant. Between pulses, the split between atomic and molecular hydrogen is determined by the quiescent electron temperature. All the appropriate rates are given in Appendix C.

\section{Discharge Dynamics}

Exploiting the fact that the tube length greatly exceeds the radius, we divide the plasma into a number of long, thin axial zones. Rate equations for the plasma constituents are set up in each zone, with the various species assumed to have a radial profile corresponding to the reciprocal of the gas temperature in that zone (cf. Sec. 4). The variation of the wall temperature with length, which is regarded as input, determines the copper density within each zone. The vapor pressure of copper was taken from measurements $[77,78]$ recommended in [79]. As expected, these data conform closely to the expression

$$
p=c_{1} \exp \left(-c_{2} / k T\right) \text {. }
$$

The coefficients are found to be $c_{1}=3.638 \times 10^{8}$ torr and $c_{2}=37020 \mathrm{~K}$.

For the bound states of copper, the rate equations have the form

$$
\dot{n}_{i}=n_{e}\left[\sum_{j \neq i}\left(n_{j} \kappa_{j i}-n_{i} \kappa_{i j}\right)-n_{i} \kappa_{i N}\right]+\sum_{j>i} n_{j} A_{j i} f_{j i}-\sum_{j<i} n_{i} A_{i j} f_{i j}+R_{i}^{I},
$$

where the index $j$ ranges over other bound states and $N$ denotes the ion. The term proportional to $n_{e}$ describes electron-impact processes, with the three inner terms corresponding to excitation/deexcitation gains, excitation/deexcitation losses, and ionization losses, respectively. The next two terms describe radiative gains and losses, respectively, with the $f$ factors accounting for radiation trapping. The latter are handled via the Holstein theory, as elaborated in Appendix D.

The term $R_{i}^{I}$, which occurs only in the rates for the lasing levels, describes the effects of optical pumping. We adopt a saturated amplifier model, according to which the upper and lower populations are clamped once the inversion begins. Denoting these densities by $n_{b}$ and $n_{a}$, respectively, we define the pumping terms such that

$$
\begin{aligned}
& \dot{n}_{a}=\frac{g_{a}}{g_{a}+g_{b}}\left[\dot{n}_{a}^{(0)}+\dot{n}_{b}^{(0)}\right], \\
& \dot{n}_{b}=\frac{g_{b}}{g_{a}+g_{b}}\left[\dot{n}_{a}^{(0)}+\dot{n}_{b}^{(0)}\right],
\end{aligned}
$$

where $\dot{n}_{a}^{(0)}$ and $\dot{n}_{b}^{(0)}$ denote all the other rates in Eq. (3), and $g_{a}$ and $g_{b}$ are the degeneracies. These conditions guarantee that, after the inversion begins $\left(n_{a} / g_{a}=n_{b} / g_{b}\right)$, the upper and 
lower populations change in synchronization $\left(\dot{n}_{a} / g_{a}=\dot{n}_{b} / g_{b}\right)$. The volume rate at which energy is lost by this process is

$$
\begin{aligned}
Q & =\sum_{i=a, b} E_{i}\left[\dot{n}_{i}^{(0)}-\dot{n}_{i}\right] \\
& =\left(E_{b}-E_{a}\right) \frac{\dot{n}_{b}^{(0)}-g_{b} \dot{n}_{a}^{(0)} / g_{a}}{1+g_{b} / g_{a}}
\end{aligned}
$$

with $E_{i}$ the energy levels. In our model, this energy is converted to axially directed photons having intensity $I=\ell Q$, with $\ell$ the length of the axial zone.

The copper ion density changes at the rate

$$
\dot{n}_{N}=n_{e} \sum_{i<N} n_{i} \kappa_{i N}-n_{e}^{2} n_{N} \kappa_{r e c}
$$

with the last term giving the three-body recombination rate.

The neon and hydrogen levels obey equations similar to (3) and (6), without the stimulated emission terms. In the case of hydrogen, there are also terms corresponding to molecular dissociation and recombination.

The rate of change of the electron temperature follows from the energy balance equation for the electron energy $U_{e}=(3 / 2) n_{e} k T_{e}$, which has the form

$$
\dot{U}_{e}=Q_{E}+Q_{\text {inel }}+Q_{e l}+Q_{\text {rec }}+Q_{P} \text {. }
$$

The first term on the right-hand side is ohmic heating,

$$
Q_{E}=E_{z}^{2} / \rho,
$$

while the second gives the effects of inelastic losses, summed over atoms,

$$
Q_{\text {inel }}=n_{e} \sum_{a} \sum_{i, j} n_{i} \kappa_{i j}\left(E_{i}-E_{j}\right) \text {. }
$$

The third term indicates equilibration with the gas temperature via elastic collisions:

$$
Q_{e l}=-n_{e}\left(T_{e}-T\right) \sum_{a} \frac{3 m_{e}}{M_{a}}\left[\kappa_{e a}^{m t}\left(T_{e}\right) n_{a}^{0}+\kappa_{e i}^{m t}\left(T_{e}\right) n_{a}^{+}\right]
$$

with $n_{a}^{0}$ and $n_{a}^{+}$denoting the density of neutrals and ions, respectively. The fourth term, which gives the heating due to recombination, has the form

$$
Q_{r e c}=\left[\Delta E_{r e c}+b\left(E_{N}-E_{N-1}-\Delta E_{r e c}\right)\right] \kappa_{r e c} n_{e}^{2} n_{N},
$$

with $\Delta E_{\text {rec }}$ indicating the energy difference between the ion ground state and the state to which recombination occurred. We assume that this is a high-n state lying above the pseudostate. As suggested by computer simulations [80], we take $\Delta E_{\text {rec }}=(3 / 2) a k T_{e}$, with $a$ of order unity. The recombining electron then decays to the pseudostate, with a fraction $b$ of the energy loss attributed to superelastic electron collisions and the remainder to spontaneous radiation. In the absence of detailed information, we take $b=1 / 2$. Finally, the term $Q_{P}$ in Eq. (7) is a small contribution due to Penning ionization. 


\section{Gas Heating Model}

The equation governing the gas energy density is, in principle,

$$
\frac{\partial}{\partial t}\left(\frac{3}{2} n T+\frac{1}{2} \rho v^{2}\right)+\nabla \cdot\left[\mathrm{v}\left(\frac{5}{2} n T+\frac{1}{2} \rho v^{2}\right)-\kappa \nabla T\right]=P,
$$

where $\mathbf{v}$ is the gas velocity, $\kappa$ is the thermal conductivity, and $P$ is the power per unit volume deposited in the gas. The power is given by a sum over momentum transfer collisions between electrons and heavy species as

$$
P=n_{e}\left(T_{e}-T\right) \sum_{a} \frac{3 m_{e}}{M_{a}}\left[\kappa_{e a}^{m t}\left(T_{e}\right) n_{a}^{0}+\kappa_{e i}^{m t}\left(T_{e}\right) n_{a}^{+}\right] .
$$

If the flow is near sonic, $v \simeq 10^{5} \mathrm{~cm} / \mathrm{s}$, disturbances propagate across the tube radius in some $10 \mu \mathrm{s}$. On this time scale the details of the temperature distribution require a treatment of gas kinetics, which is beyond the scope of our model. On a longer time scale, such as the interpulse period, convective disturbances will have damped out, so that one can consider the steady state conduction equation

$$
\nabla \cdot(\kappa \nabla T)=-\bar{P}
$$

with $\bar{P}$ the time-averaged power deposited in a particular axial zone. This is the model employed here. In addition, we make the reasonable assumption that the power is deposited uniformly across the discharge cross section. Of course the deposited power density varies from one axial zone to another, since the plasma constituents also vary.

In practice, the code constructs a steady state temperature distribution by following a sequence of several pulses. In the first pulse, the gas temperature is simply taken as the wall temperature. At the end of this pulse, the time-averaged deposition is used to solve for a new temperature distribution, via the last equation. The spatial average of this is then used as the temperature during the second pulse, with a new distribution calculated at the end, and so forth. Convergence within $0.1 \%$ is usually obtained after three or four pulses.

The average temperature is to be weighted with the gas density, i.e. $\bar{T}=\langle n T\rangle \mid\langle n\rangle$. From the ideal gas law, this means that the appropriate average temperature is

$$
\bar{T}=\left\langle T^{-1}>^{-1}\right. \text {. }
$$

The thermal conductivity of neon is given quite accurately by [8]

$$
\kappa=\kappa_{0} T^{m},
$$

with $m=0.639$ and and $\kappa_{0}=1.274 \times 10^{-3} \mathrm{~W} /\left(\mathrm{m} \mathrm{K}^{\mathrm{m}+1}\right)$. In the temperature range 1400$3000 \mathrm{C}$, the thermal conductivity increases from about 0.14 to $0.22 \mathrm{~W} / \mathrm{mK}$.

More generally, it is convenient to write the thermal conductivity in the form $\kappa(T)=$ $\mu^{\prime}(T)$, so that the heat conduction equation becomes simply

$$
\nabla^{2} \mu=-\bar{P} \text {. }
$$

After solving this equation for $\mu$, we obtain the temperature distribution by applying the inverse of $\mu$. We proceed to show how this is done in the cases of interest. 


\subsection{Cylindrical Laser}

Expanding the Laplacian and imposing the boundary conditions $T(a)=T_{w}$ and $T^{\prime}(0)=0$, we find

$$
\mu[T(r)]=\mu\left(T_{w}\right)+\frac{1}{4} \bar{P}\left(a^{2}-r^{2}\right) .
$$

The temperature distribution in neon therefore has the form [7]

$$
T(r)=T_{w}[1+\alpha(1-x)]^{1 /(m+1)},
$$

where $x=(r / a)^{2}$ and $\alpha$ is a dimensionless parameter proportional to the deposited power per unit length:

$$
\alpha=\frac{\bar{P} a^{2}}{4 \mu\left(T_{w}\right)}=\frac{(m+1) \bar{P} a^{2}}{4 \kappa_{0} T_{w}^{m+1}} .
$$

Typically one has $1<\alpha<2$. The average temperature is then

$$
\bar{T}=G_{m}^{(c y l)}(\alpha) T_{w}
$$

with

$$
G_{m}^{(c y l)}(\alpha)=\frac{\alpha m}{m+1}\left[(1+\alpha)^{m /(m+1)}-1\right]^{-1} .
$$

The temperature enhancement factor $G_{m}^{(c y l)}$ is plotted as a function of the deposition in Fig. 2. For typical values of $\alpha$, it ranges from about 1.2 to 1.5. A sample temperature profile is shown in Fig. 3. Note that the temperature rises from $1490 \mathrm{C}$ at the wall to about $2900 \mathrm{C}$ at the center.

\subsection{Septum Laser}

We turn to the heat conduction equation, with a 2-dimensional temperature distribution $T(r, \theta)$. The angle is measured with respect to a vertical section from the septum, as shown in Fig. 4. We assume that the radiative coupling is sufficiently strong to force the septum to have the same temperature as the walls. Estimates for a molybdenum septum, with appropriate radiation view factors and emissivities, show that the septum is about $70 \mathrm{C}$ hotter than the wall [9]. Since the difference is only a few percent of the gas temperature, it does not represent a significant effect.

We expand $\mu$ in a Fourier series with respect to $\theta$, noting that only cosine terms can contribute because of symmetry:

$$
\mu[T(r, \theta)]=\mu\left(T_{w}\right)+\sum_{\ell=0}^{\infty} a_{\ell}(r) \cos (\ell \theta) .
$$

It is also clear that $\ell$ will be limited to odd values, in order to enforce the boundary condition that $T=T_{w}$ along the septum $(\theta= \pm \pi / 2)$. The solution works out to be

$$
T(r, \theta)=\mu^{-1}\left[\mu\left(T_{w}\right)+\frac{4 \bar{P} a^{2}}{\pi} \sum_{\ell \text { odd }} \frac{\left(u^{2}-u^{\ell}\right)}{\ell\left(\ell^{2}-4\right)}(-1)^{(\ell-1) / 2} \cos (\ell \theta)\right],
$$


with $u=r / a$.

Inserting the specific form of the neon thermal conductivity, we have

$$
\bar{T}=T_{w} G_{m}^{(s)}(\alpha)
$$

in terms of the function

$$
G_{m}^{(s)}(\alpha)=\left[\frac{4}{\pi} \int_{0}^{1} d u u \int_{0}^{\pi / 2} d \theta\left(1+\frac{16 \alpha}{\pi} \sum_{\ell \text { odd }} \frac{\left(u^{2}-u^{\ell}\right)}{\ell\left(\ell^{2}-4\right)}(-1)^{(\ell-1) / 2} \cos (\ell \theta)\right)^{-1 /(m+1)}\right]^{-1} .
$$

This function is plotted in Fig. 2. A typical temperature profile, for the same deposition as in the cylindrical case, is plotted in Fig. 3. Note that the maximum temperature is now only $2100 \mathrm{C}$, about $800 \mathrm{C}$ below that of the cylindrical case.

\section{Electromagnetics}

In this section, we obtain the equations for the driving laser fields, in a realistic onedimensional approximation. A schematic drawing of the laser assembly is shown in Fig. 5 . The salient features are that the plasma is cylindrical, that it is surrounded radially by insulating regions having various permittivities, that the assembly is enclosed in a metal shell except for gaps at which wires leading back to the modulator are attached, and that the entire assembly is rotationally symmetric. Of course the insulator-plasma interface must be straight.

We allow the permittivity of the insulator to be an arbitrary function of $r$ and $z$. We take the conductivity of the insulator to be zero and that of the metal to be infinite.

We ignore the displacement current in Maxwell's equations, since it gives rise to field variations having time scales shorter than those of interest (nanoseconds to hundreds of microseconds). This is seen as follows. In conductors, the displacement current leads to time scales of order $\tau \simeq \epsilon / \sigma$. For a typical copper plasma, this is in the neighborhood of $10^{-14} \mathrm{~s}$. In insulators the displacement current is relevant for times of order $\ell / c$ ( $\ell$ a typical dimension), which is generally less than a nanosecond.

In cylindrical geometry, the solutions of Maxwell's equations divide into transverse magnetic $\left(B_{z}=0\right)$ and transverse electric $\left(E_{z}=0\right)$ modes. The former mode is appropriate for our case. Since the fields are independent of $\theta$, the only nonvanishing field components are $B_{\theta}, E_{r}$, and $E_{z}$. Maxwell's equations then consist of the $r$-component and $z$-component of Ampere's law,

the $\theta$-component of Faraday's law,

$$
\begin{gathered}
\frac{\partial B}{\partial z}=-\mu_{0} J_{r}, \\
\frac{1}{r} \frac{\partial(r B)}{\partial r}=\mu_{0} J_{z},
\end{gathered}
$$

$$
\frac{\partial B}{\partial t}=\frac{\partial E_{z}}{\partial r}-\frac{\partial E_{r}}{\partial z}
$$


and Gauss's law,

$$
\frac{1}{r} \frac{\partial\left(r \epsilon E_{r}\right)}{\partial r}+\frac{\partial\left(\epsilon E_{z}\right)}{\partial z}=\rho .
$$

Here $\rho$ is the free charge and $B$ stands for $B_{\theta}$. In Ampere's law, the current is given by $\mathbf{J}=\mathbf{E} / \eta$, with $\eta$ the resistivity. The divergence of the magnetic field vanishes automatically since $B_{\theta}=B_{\theta}(r, z)$.

These equations are treated differently, depending on whether we are dealing with the plasma or with insulating regions.

\subsection{Fields within Plasma}

Within the plasma, we use the fact that the conductivity is nonzero and obtain the electric field from Ampere's law. Inserting it into Faraday's law, we have the following equation for the magnetic field:

$$
\frac{\partial B}{\partial t}=\frac{\partial}{\partial r} \frac{D}{r} \frac{\partial(r B)}{\partial r}+\frac{\partial}{\partial z} D \frac{\partial B}{\partial z},
$$

where $D=\eta / \mu_{0}$. This is a two-dimensional diffusion equation, with diffusion coefficient $D$. Note that the electric field can be obtained in terms of $B$ via

$$
\begin{gathered}
E_{r}=-D \frac{\partial B}{\partial z}, \\
E_{z}=\frac{D}{r} \frac{\partial(r B)}{\partial r} .
\end{gathered}
$$

Taking into account the simplification that the laser length far exceeds its width, we divide the plasma into a number of long axial zones, as noted in Sec. 3. We expect the magnetic field to vary slowly within each zone and the electric field to be directed primarily in the $z$ direction. At the boundary between zones, however, we expect there to exist a transition layer of width comparable to the plasma radius. Within this layer, the magnetic field should interpolate between its values in the bulk of the zones and the electric field should develop a radial component. If the zones are long compared to the width of the transition layer, we can idealize this situation by ignoring the transition layer. Then in each zone $i$ the magnetic field $B_{i}(r, t)$ obeys the one-dimensional diffusion equation

$$
\frac{\partial B_{i}}{\partial t}=\frac{\partial}{\partial r} \frac{D_{i}}{r} \frac{\partial\left(r B_{i}\right)}{\partial r},
$$

with $D_{i}=\eta_{i} / \mu_{0}$ within the zone. At the radial boundary $r=a$, the field must correspond to an enclosed current equal to the plasma current, or

$$
B_{i}(a, t)=\frac{\mu_{0}}{2 \pi a} I_{p}(t) .
$$

This holds for each axial zone, since the plasma current is conserved. At the center the magnetic field vanishes. 
We obtain an additional piece of information by taking the surface integral of Faraday's law around the $r-z$ cross section of the insulating region. This yields the following relation between the loop voltage and the derivative of the plasma current:

$$
V_{\ell}-\sum_{i} \ell_{i} E_{z, i}(a, t)=L_{c} \frac{d I_{p}}{d t}
$$

where $V_{\ell}$ is the voltage across the laser assembly (i.e. across the peaking capacitor and its effective series resistor), $\ell_{i}$ is the length of a zone, and $L_{c}$ is the inductance associated with the insulator,

$$
L_{c}=\frac{\mu_{0}}{2 \pi} \int r^{-1} d r d z
$$

Here the integral extends over the insulating cross section in the $r-z$ plane. In this approximation, the various zones can be situated in any order.

\subsection{Fields within Insulator}

Since the insulator carries no current, the magnetic field is given by the enclosed plasma current. via

$$
B(r, z, t)=\frac{\mu_{0}}{2 \pi r} I_{p}(t),
$$

independently of $z$. Given the magnetic field, we solve for the electric field by combining Gauss's law (with no free charge) and Faraday's law. An efficient way of doing this is to note that, since $\operatorname{div}(\epsilon \mathbf{E})=0$, we can represent $\epsilon \mathbf{E}$ in terms of a vector potential as $\epsilon \mathbf{E}=\nabla \times \mathbf{C}$, so that

$$
\begin{gathered}
\epsilon E_{r}=-\frac{\partial C_{\theta}}{\partial z}, \\
\epsilon E_{z}=\frac{1}{r} \frac{\partial\left(r C_{\theta}\right)}{\partial r} .
\end{gathered}
$$

Then Faraday's law becomes the following static equation for $\varphi \equiv r C_{\theta}$ :

$$
r \frac{\partial}{\partial r} \frac{1}{\epsilon r} \frac{\partial \varphi}{\partial r}+\frac{\partial}{\partial z} \frac{1}{\epsilon} \frac{\partial \varphi}{\partial z}=\frac{\mu_{0}}{2 \pi} \frac{d I_{p}}{d t} .
$$

This is an elliptic equation, with boundary conditions determined as follows. First, at the boundary with the plasma, $E_{z}$ is continuous and so we have a condition on the radial derivative of $\varphi$. Second, at a boundary with the metal, the tangential component of the electric field must vanish. This means that

$$
\begin{array}{cc}
\frac{\partial \varphi}{\partial r}=0 & \text { (horizontal boundary) } \\
\frac{\partial \varphi}{\partial z}=0 & \text { (vertical boundary) }
\end{array}
$$

These are Neumann boundary conditions. Hence Eq. (41) determines $\varphi$ within an additive constant, and the electric field is determined uniquely. 
We can see as follows that charge builds up at the interface between the plasma and the insulator. We construct a narrow container which encloses this interface and which encircles the plasma, and integrate Gauss's law within this container. There is a contribution to the surface integral only within the insulator, since $E_{z}=0$ just within the plasma. Hence the surface charge density is

$$
\Sigma=\epsilon E_{z},
$$

so that it is proportional to the normal component of the electric field within the insulator. Actually this charge builds up on the time scale $\epsilon / \sigma$.

In summary, the insulator fields can be found, at convenient times of interest, by means of a statics calculation. It is important to note that the fields within the plasma can be found without solving for the fields within the insulator.

\subsection{Plasma Resistivity}

Neglecting convective terms and pressure gradients, electron momentum balance reads

$$
e n_{e}\left(\mathbf{E}+\mathbf{u}_{\mathbf{e}} \times \mathbf{B}\right)=\mathbf{R}_{e}, \quad(e>0)
$$

where $\mathbf{R}_{e}$ is the collisional friction between the electrons and other constituents. This breaks up into an electron-ion contribution and a sum over the electron-atom contributions:

$$
\mathbf{R}_{e}=\mathbf{R}_{e i}+\sum_{a} \mathbf{R}_{e a} \text {. }
$$

Each term is the first velocity moment of the appropriate Boltzmann collision integral, e.g. for electron-atom collisions:

$$
\mathbf{R}_{e a}=m_{e} \int d^{3} v \mathbf{v} C_{e a} .
$$

Following Braginskii [81], we assume a Maxwellian atom distribution function. We expand the electron distribution function as a Maxwellian corrected by a drift velocity $u_{e}$. The electron-atom friction then reduces to

$$
\mathbf{R}_{e a}=-n_{a} n_{e} m_{e} \kappa_{e a} \mathbf{u}_{e}
$$

in terms of the rate coefficient

$$
\kappa_{e a}=\frac{m_{e}}{3 T_{e}}<\sigma_{e a}^{m t} v^{3}>,
$$

with $\sigma^{m t}$ the momentum transfer cross section. Note that this differs from the usual $\langle\sigma v\rangle$ in that the average contains two additional powers of $v$. One factor follows from the fact that this is a momentum transfer process, and the other factor follows from the fact that only the part of the electron distribution function proportional to $\mathbf{v} \cdot \mathbf{u}_{\mathbf{e}}$ contributes to the rate. The extra powers of $v$ are normalized by the thermal speed.

The electron-ion friction, projected in the direction perpendicular to the field, has the form [81]

$$
\mathbf{R}_{e i}=-m_{e} n_{e} \nu_{e i}\left(1-\ell^{\perp}\right)\left(\mathbf{u}_{e}-\mathbf{u}_{i}\right)_{\perp}+m_{e} n_{e} \Omega \ell^{\wedge} \mathbf{n} \times\left(\mathbf{u}_{e}-\mathbf{u}_{i}\right)
$$


with $\nu_{e i}$ the electron-ion $90^{\circ}$ collision frequency, $\Omega$ the electron gyrofrequency, and $\mathbf{n}$ a unit vector in the direction of $\mathbf{B}$. The quantities $\ell^{\perp}$ and $\ell^{\wedge}$ are functions of the ratios of the collision frequencies to the gyrofrequency. This component of the friction is all that we require.

Taking the $r$ and $z$ components of the last equation, inserting the expressions for the friction terms, and ignoring the radial electric field, we obtain Ohm's law in the form $J_{z}=$ $E_{z} / \eta$, with the resistivity given by

$$
\eta=\frac{m_{e} \nu}{e^{2} n_{e}}\left[1+\frac{\Omega^{2}}{\nu \nu^{\prime}}\left(1+\ell^{\wedge}\right)\right]
$$

with $\nu$ the total electron collision frequency (i.e. with both ions and atoms) and $\nu^{\prime}$ the electron-atom collision frequency:

$$
\nu=\left(1-\ell^{\perp}\right) \nu_{\mathrm{ei}}+\nu^{\prime}, \quad \nu^{\prime}=\sum_{a} n_{\mathrm{a}} \kappa_{e a}^{m t}
$$

The part of $\eta$ proportional to $\Omega^{2}$ is the enhancement of the resistivity due to the magnetic field. In typical copper lasers this usually is no more than $5 \%$.

\section{Computational Scheme}

The variables of the final model consist of the densities of the atomic states, the extracted laser intensity, the magnetic field of the laser, and the modulator variables (voltages, currents, and the fields of the magnetic switches). The modeling of the modulator, a significant problem in itself, is discussed separately $[69,83,84]$. Geometrically, the atomic and laser variables depend on the axial zone, with the dependence entering parametrically via the local wall temperature. The magnetic field depends on both $r$ and the axial zone, via the field diffusion equation (34). We difference this equation in $r$, obtaining equations for the evolution $B$ at discrete points.

The model thus reduces to a system of ordinary differential equations in time. In a typical problem with five axial zones, driven by the Laser Demonstration Facility modulator [69, 82], there are 90 atomic and laser variables (seven copper states, three neon states, five hydrogen states, the electron temperature, and the two intensity colors, all indexed by the axial zone), 45 magnetic field variables (with 9 radial solution points), and 15 modulator variables, for a total of 150 variables.

We treat all the variables in a unified computational manner, by advancing them in time with an ODE solver. The system is mathematically stiff. We employ the ODE solver LSODKR, a variant of the standard LSODE package [85]. This solver employs Krylov methods in numerical iterations and has root-finding capabilities which are helpful in treating the extracted intensity and the magnetic switches. Details of these methods are discussed in [86] and [87]. 


\section{Results}

We now use the model to calculate performance predictions for the laser amplifier with septum inserts. This device has a radius of $4 \mathrm{~cm}$, a tube length of $273 \mathrm{~cm}$, and a septum insert. It is driven by the Laser Demonstration Facility modulator mentioned above [69, 82]. The simulations presented here do not include the effects of hydrogen. Some results of the model with hydrogen are given in [9].

The laser model requires the plasma tube temperature profile along the length of the tube. This was taken from TOPAZ calculations by A. T. Anderson [88]. A typical profile is shown in Fig. 6. Note that this figure shows only half the length of the tube, since the temperature is symmetrical about the midpoint. The half-tube has been divided into five axial zones. The lengths of these zones are set so that the average copper density at the wall, also shown in Fig. 6, changes in approximately uniform steps.

Figure 7 shows predictions for the lasing intensities, at standard operating conditions $\left(V_{0}=18 \mathrm{kV}, p=40\right.$ torr $)$. Since the ${ }^{2} \mathrm{D}$ and ${ }^{2} \mathrm{P}$ densities, as plotted, are divided by their degeneracies, the curves touch during the amplification interval. In the second part of this figure, the calculated extracted intensity is compared with measurements by J. J. Chang [89]. The curves have similar shapes, rising quickly and falling relatively slowly over a period of about $70 \mathrm{~ns}$. The predicted power is $428 \mathrm{~W}$, at a pulse repetition rate of $4.3 \mathrm{kHz}$, as compared to the measured power of approximately $300 \mathrm{~W}$.

Now we explore the full range of experimental voltages and pressures by collating a large number of runs. Several hundred runs were made to cover the entire space of input parameters (driving voltage, gas pressure, and wall temperature), at the same pulse repetition rate as above. In the runs, the central wall temperature ranged from $1375 \mathrm{C}$ to $1600 \mathrm{C}$, in steps of $25 \mathrm{C}$. The selected gas pressure ranged from 10 to 110 torr, in steps of 10 torr; and the voltage varied from 16 to $22 \mathrm{kV}$, in steps of $0.5 \mathrm{kV}$. Thus runs were made at $10 \times 11 \times 15=1430$ points.

The resulting laser power is plotted as a function of central wall temperature, for selected voltages and pressures, in Fig. 8. These plots show that, at a given voltage, both the optimum wall temperature and the maximum power increase with pressure. At $18 \mathrm{kV}$, for example, the optimum wall temperature increases from about $1425 \mathrm{C}$ (at 10 torr), to about $1500 \mathrm{C}$ (at 110 torr). At the same time, the maximum power increases from $300 \mathrm{~W}$ to $650 \mathrm{~W}$. At a given pressure, on the other hand, neither the optimum wall temperature nor the maximum power is very sensitive to voltage. Thus at a pressure of 30 torr, as the voltage is raised from 16 to $19 \mathrm{kV}$, the optimum wall temperature ranges from about $1450 \mathrm{C}$ to $1475 \mathrm{C}$. The corresponding maximum power increases from $330 \mathrm{~W}$ to only $430 \mathrm{~W}$.

The main effect of the voltage is to control the power absorbed in the wall, i.e. the ohmic heating in the plasma minus the laser power. Figure 9 shows plots of the absorbed power per unit length, halfway along the length of the tube, versus wall temperature for the same choices of pressure and voltage as above. At given pressure and voltage, the absorbed power is practically independent of the wall temperature. At fixed pressure, however, the absorbed power increases by about $30 \%$ as the voltage is raised from $16 \mathrm{kV}$ to $19 \mathrm{kV}$.

To visualize the detailed dependencies of these results, it is convenient to employ con- 
tour plots. This is done via a postprocessor code, which collates and analyzes results from a collection of runs. The first plot in Fig. 10 shows contours of the laser power on the temperature-pressure plane, for a fixed voltage of $17 \mathrm{kV}$. One sees that the contours form a "mountain", with a peak of about $600 \mathrm{~W}$ for moderate temperatures (about $1500 \mathrm{C}$ ) and pressures of 100 torr or greater. Similarly, the second plot in Fig. 10 shows power contours on the temperature-voltage plane, for a fixed pressure of 70 torr. Here again a mountain structure is evident for moderate temperatures and high voltages.

Not all points on these contour plots are accessible in experiment, since the wall temperature is not controlled independently. Instead, it is determined by the absorbed power and the characteristics of the particular thermal package. In particular, the central wall temperature is a function of the central absorbed power per unit length, say $T=F\left(P_{a b s}\right)$. This function can be estimated both from calculations and from calorimetry. Although substantial uncertainties exist, a TOPAZ calculation by A. T. Anderson [88] appears to be realistic and is employed here. It is shown in Fig. 11.

The absorbed power, as calculated by the model, is a function of wall temperature, pressure, and voltage, say

$$
P_{a b s}=P_{a b s}(T, p, V) \text {. }
$$

At a given pressure and voltage, then, the operational wall temperature is determined as the root of

$$
T=F\left[P_{a b s}(T, p, V)\right] \text {. }
$$

To handle this equation numerically, we fit the absorbed power to a polynomial of the following form in $T, p$, and $V$ :

$$
P_{a b s}(T, p, V)=\sum_{i_{1}=1}^{N 1} \sum_{i_{2}=1}^{N_{2}} \sum_{i_{3}=1}^{N_{3}} A_{i_{1}, i_{2}, i_{3}} T^{i_{1}-1} p^{i_{2}-1} V^{i_{3}-1}
$$

It was found sufficient to take a quadratic in $T$ and quartics in $p$ and $V$ (i.e. $N_{1}=3$, $N_{2}=N_{3}=5$ ). The wall temperature was then found via a root-solving routine.

Using this form for the wall temperature, and returning to Fig. 10, we see that the system passes along a trajectory on the temperature-voltage plane, as the absorbed power increases. The trajectory is indicated by the dotted line. Note that it ascends the power "mountain" only partially before sliding around and descending the opposite side. A similar trajectory occurs on the temperature-voltage plane. The laser power encountered along these trajectories, plotted in Fig. 12, peaks between 450 and $500 \mathrm{~W}$. To increase the power, one should employ a thermal package which absorbs increased power at a given wall temperature.

Putting all this information together, we show in Fig. 13 the predicted wall temperature and laser power as a function of pressure and voltage, for the particular thermal package. To maintain a fixed wall temperature, one would decrease the pressure and increase the voltage (or vice versa) along a contour. For the laser power, there is a ridge extending from low pressures and high voltages to high pressures and low voltages. The high power regime, which occurs at the latter end of the ridge, is in the neighborhood of $16 \mathrm{kV}$ and 100 torr. The power obtained there, about $500 \mathrm{~W}$, is more than twice that found at the low end of the ridge. 
Experimentally, the maximum power occurs at about this point, but it varies less strongly with position on the plane. The maximum experimental power is about 300-350 W. Thus the model prediction is about $45 \%-65 \%$ high.

Generally speaking, one would expect the model to overestimate the extracted power, since it does not include factors such as misalignment and dirty windows which inevitably occur in experiment. In addition to these, two plasma physics factors could be playing a role in this discrepancy. First, the voltage fall at the cathode should lead to an increased power absorption and thus a distortion of the mountain structure. A primitive model of this effect has been implemented in the code, but it was not employed in the present runs because of uncertainties regarding the parameters of the model and, indeed, the validity of the model itself. A second factor could be radial transport. As discussed earlier, the magnetic field is allowed to diffuse radially, but the plasma constituents are treated as having a radial profile determined by the gas temperature. Since the discharge is heated from the outside, inclusion of radial transport would be expected to decrease the extracted power.

\section{Conclusions}

We have described a detailed computational model of a copper laser amplifier. We have presented rate equations for the constituent species, along with equations for the electron temperature, the extracted laser intensity, and the diffusing magnetic field. These equations are coupled to the model of the driving modulator by the boundary value of the magnetic field, which gives the plasma current. Rates have been constructed for all appropriate atomic reactions. The radial profile of the gas temperature is determined by the time-averaged power deposited in the gas. We have taken into account the role of septum inserts in cooling the gas.

We have used the model, in conjunction with a modulator model, to calculate comprehensive performance predictions for a high-power septum laser amplifier. This analysis included both a representative operating point and a systematic survey of operating points. In general, the calculated laser power appears to lie about $50 \%$ above experiment. Some reasons for this discrepancy were outlined.

To refine the model, one would begin by comparing its predictions with more detailed experimental information, such as the line-averaged densities of the ground [8] and excited $[62,63]$ states. This would also involve the addition of radial transport. A great deal of this work has actually been done, but attention has subsequently shifted to other projects.

In related work, the model has been used in conjunction with a model of pulse extraction from an amplifier [90]. Here the saturation assumption is removed, and the pulse height and lasing densities are calculated as functions of axial position and time. For a laser without a septum, the saturated power agrees with experiment and the results at lower powers show the expected trend.

In summary, we believe that our model contains the most important physics underlying the copper laser. It has proved to be a very useful tool for understanding copper lasers in the laboratory. 


\section{Acknowledgments}

We would like to thank E. R. Ault, J. J. Chang, A. A. Mondelli, and P. D. Weber for discussions. We are indebted to K. F. Scheibner and A. U. Hazi for the cross section calculations of [99] and for discussions of related atomic physics issues. We are also indebted to A. C. Hindmarsh, who provided the ODE solver and worked with us to determine the optimal manner of applying it to the problem. Finally, we are grateful to G. D. Duckworth for skilled computational assistance. 


\section{A Copper Rates}

\section{A.1 Radiative Transition Rates}

A critical survey of transition rates for the observed spectral lines of $\mathrm{CuI}$ has been presented by Bielski [91]. Matching his cited wavelengths with energy spacings given by Moore [73], one can identify a total of 42 observed transitions pertinent to our model. These begin within or below the pseudostate and terminate on the ground state or the lasing levels. In addition, three ultraviolet transitions probably are significant but are not listed. These are the pair of transitions

$$
(5 p){ }^{2} \mathrm{P}_{3 / 2,1 / 2}^{o} \rightarrow(4 s){ }^{2} \mathrm{~S}_{1 / 2},
$$

and also

$$
(5 \mathrm{p})^{2} \mathrm{P}_{3 / 2}^{\circ} \rightarrow\left(4 \mathrm{~s}^{2}\right)^{2} \mathrm{D}_{3 / 2} \text {. }
$$

The first two transitions are similar to the resonance transitions except that the initial configuration is $5 p$ instead of $4 p$. For lack of better information, we estimate their oscillator strengths as half that of the corresponding resonance lines. The last transition, which has an initial $\mathrm{J}$ of $3 / 2$, has been observed for an initial $\mathrm{J}$ of $1 / 2$. We assume that the rates for the two $\mathrm{J}$ values have the same proportion as the rates for the two similar lines

$$
\text { (4p) }{ }^{2} \mathrm{P}_{3 / 2,1 / 2}^{o} \rightarrow\left(4 \mathrm{~s}^{2}\right)^{2} \mathrm{D}_{3 / 2} \text {. }
$$

All 45 lines are listed in Table 4.

Five of the lines shown in the table connect states below the pseudostate. These are the pair of resonance lines $(327.39 \mathrm{~nm}$ and $324.75 \mathrm{~nm})$, the two lasing lines, and also the weak line connecting the $J=3 / 2$ components of the lasing states (cf. reaction above). Note that the resonance lines are stronger than the lasing lines by nearly two orders of magnitude. There is no radiation from the metastable states.

Since the populations of the individual members of the pseudostate are not followed as functions of time, it is necessary to average the radiation from these levels to the ground state and the lasing states. Averaging the rates over statistical weights appropriate to local thermodynamic equilibrium, we obtain effective $T_{e}$-dependent rates to the five lower states. These rates, designated $A_{p i}\left(T_{e}\right)(i=1, \ldots, 5)$, are shown in Fig. 14.

\section{A.2 Excitation}

The electron-impact excitation cross sections of copper are difficult to measure. Early data for the transitions $(4 \mathrm{~s})^{2} \mathrm{~S} \rightarrow(4 \mathrm{p})^{2} \mathrm{P}$ and $(4 s)^{2} \mathrm{~S} \rightarrow\left(4 \mathrm{~s}^{2}\right)^{2} \mathrm{D}$ were obtained by Williams and Trajmar [92] and by Trajmar et al. [93], with the second reference superseding the first. A change in the absolute normalization of the results was suggested on theoretical grounds in [94]. The resonance cross section was also measured by Aleksakhin et al. [95], with results which tend to confirm this renormalization. In addition, a point at $60 \mathrm{eV}$ was recently obtained by Teubner [96]. All of these experiments were in the energy range of 6-100 eV, which only marginally overlaps the regime of interest. 
Table 4: Copper Radiative Transitions in the Model

\begin{tabular}{|c|c|c|c|}
\hline initial state & final state & $\lambda(\mathrm{nm})$ & $A\left(10^{7} s^{-1}\right)$ \\
\hline$(4 p)^{2} P_{1 / 2}^{0}$ & $(48)^{2} S_{1 / 2}$ & $\mathbf{3 2 7 . 3 9}$ & 13.6 \\
\hline$(4 p)^{2} P_{3 / 2}^{0}$ & $\Rightarrow$ & 324.75 & 13.7 \\
\hline$\left(4 p^{\prime}\right)^{4} \mathrm{P}_{3 / 2}^{0}$ & $\Rightarrow$ & 249.21 & .306 \\
\hline$\left(4 p^{\prime}\right)^{4} \mathrm{P}_{1 / 2}^{0}$ & $\Rightarrow$ & 244.16 & .185 \\
\hline$\left(4 p^{\prime}\right)^{4} D_{3 / 2}^{\circ}$ & 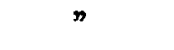 & 224.43 & .23 \\
\hline$\left(4 p^{\prime}\right)^{4} D_{1 / 2}^{\circ}$ & $”$ & 222.57 & 4.8 \\
\hline$\left(4 p^{\prime}\right)^{2} P_{1 / 2}^{0}$ & $"$ & 218.18 & 11.18 \\
\hline$\left(4 p^{\prime}\right)^{2} p_{3 / 2}^{\circ}$ & " & 217.90 & 9.4 \\
\hline$\left(4 p^{\prime}\right)^{2} D_{3 / 2}^{\circ}$ & 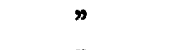 & 216.51 & 5.86 \\
\hline$(5 p)^{2} \mathrm{P}_{3 / 2}^{\circ}$ & ” & 202.438 & 18. (a) \\
\hline$(5 p)^{2} \mathrm{P}_{1 / 2}^{0}$ & $\Rightarrow$ & 202.437 & 18. (a) \\
\hline$(4 \mathrm{p})^{2} \mathrm{P}_{3 / 2}^{0}$ & $\left(4 \mathrm{~s}^{2}\right)^{2} \mathrm{D}_{5 / 2}$ & 510.54 & .195 \\
\hline$\left(4 p^{\prime}\right)^{4} P_{5 / 2}^{0}$ & 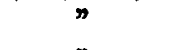 & 359.40 & .0008 \\
\hline$\left(4 p^{\prime}\right)^{4} P_{3 / 2}^{\circ}$ & $n$ & 345.78 & .0064 \\
\hline$\left(4 p^{\prime}\right)^{4} F_{T / 2}^{\circ}$ & $n$ & 333.78 & .038 \\
\hline$\left(4 p^{\prime}\right)^{4} F$ & $"$ & 329.28 & .006 \\
\hline$\left(4 p^{\prime}\right)^{4} D_{7 / 2}^{\circ}$ & $n$ & 309.40 & .0296 \\
\hline$\left(4 p^{\prime}\right)^{4} D_{5 / 2}^{\circ}$ & $”$ & 301.08 & .13 \\
\hline$\left(4 p^{\prime}\right)^{4} D_{3 / 2}^{0}$ & $"$ & 299.84 & .0027 \\
\hline$\left(4 p^{\prime}\right)^{2} F_{s / 2}^{\circ}$ & $n$ & 307.38 & .015 \\
\hline$\left(4 p^{\prime}\right)^{2} F_{7 / 2}^{0}$ & 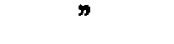 & 296.11 & .376 \\
\hline$\left(4 p^{\prime}\right)^{2} P_{3 / 2}^{\circ}$ & » & 288.29 & .18 \\
\hline$\left(4 p^{\prime}\right)^{2} D_{3 / 2}^{o}$ & $"$ & 285.87 & .0067 \\
\hline$\left(4 p^{\prime}\right)^{2} D_{3 / 2}^{\circ}$ & 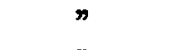 & 282.43 & .783 \\
\hline$(5 p)^{2} \mathrm{P}_{3 / 2}^{0}$ & 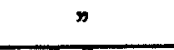 & 261.84 & 3.07 \\
\hline$(4 p)^{2} \mathrm{P}_{1 / 2}^{0}$ & $\left(48^{2}\right)^{2} D_{3 / 2}$ & 578.20 & .19 \\
\hline$(4 p)^{2} \mathrm{P}_{3 / 2}^{0}$ & 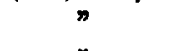 & 570.01 & .025 \\
\hline$\left(4 p^{\prime}\right)^{4} P_{3 / 2}^{0}$ & 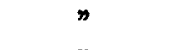 & 372.07 & .0011 \\
\hline$\left(4 p^{\prime}\right)^{4} \mathrm{P}_{1 / 2}^{\circ}$ & 》 & 360.92 & .0036 \\
\hline$\left(4 p^{\prime}\right)^{4} F_{5 / 2}^{0}$ & $”$ & 353.03 & .0262 \\
\hline$\left(4 p^{\prime}\right)^{4} D_{5 / 2}^{0}$ & ” & 320.82 & .0493 \\
\hline$\left(4 p^{\prime}\right)^{4} D_{3 / 2}^{\circ}$ & $n$ & 319.41 & .155 \\
\hline$\left(4 p^{\prime}\right)^{4} D_{1 / 2}^{\circ}$ & 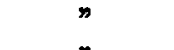 & 315.66 & .0176 \\
\hline$\left(4 p^{\prime}\right)^{2} F_{5 / 2}^{0}$ & $n$ & 327.98 & .07 \\
\hline$\left(4 p^{\prime}\right)^{2} P_{1 / 2}^{\circ}$ & $"$ & 306.89 & .0019 \\
\hline$\left(4 p^{\prime}\right)^{2} P_{3 / 2}^{\circ}$ & $"$ & 306.34 & .155 \\
\hline$\left(4 \mathrm{p}^{\prime}\right)^{2} \mathrm{D}_{3 / 2}^{\circ}$ & 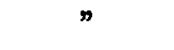 & 303.61 & .243 \\
\hline$\left(4 p^{\prime}\right)^{2} D_{5 / 2}^{\circ}$ & $"$ & 299.73 & .116 \\
\hline$(5 p)^{2} \mathrm{P}_{3 / 2}^{\circ}$ & " & 276.638 & $.1(a)$ \\
\hline$(5 p)^{2} \mathrm{P}_{1 / 2}^{\circ}$ & & 276.635 & .96 \\
\hline$(58)^{2} S_{1 / 2}$ & $(4 p)^{2} P_{1 / 2}^{\circ}$ & 793.29 & 2.250 \\
\hline$(4 d)^{2} D_{3 / 2}$ & & 515.31 & 10.340 \\
\hline$(58)^{2} S_{1 / 2}$ & $(4 p)^{2} \mathrm{P}_{3 / 2}^{0}$ & 809.24 & 4.590 \\
\hline (4d) ${ }^{2} \mathrm{D}_{3 / 2}$ & , & 522.00 & 2.180 \\
\hline$(4 d)^{2} D_{5 / 2}$ & 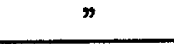 & 521.81 & 12.220 \\
\hline
\end{tabular}

(a) Estimated (see text). 
Excitation cross sections in copper were first calculated by Msezane and Henry [97], who employed a three-state basis below $10 \mathrm{eV}$ and a four-state basis from 6 to $100 \mathrm{eV}$. Later, Scheibner, Hazi, and Henry [98] undertook a calculation of the cross sections in an energy range extending down to $0.1 \mathrm{eV}$, working with a 4-state basis. Scheibner and Hazi subsequently performed a calculation with a 10-state basis Sche88a (cf. also [100]), resulting in the most comprehensive available set of excitation cross sections. All of these calculations lie below the original data [93] by as much as a factor of five, but are closer (within a factor of two) to the renormalized data.

Very recently, a measurement of the resonance excitation cross section in the low-energy regime (threshold to $8 \mathrm{eV}$ ) has been carried out [101]. The results agree within $10 \%$ with the 10-state calculations of Scheibner and Hazi.

Fortified by this agreement, we have taken the bulk of our excitation cross sections from the Scheibner-Hazi calculation. The ten basis states employed by them are $(4 \mathrm{~s}){ }^{2} \mathrm{~S},\left(4 \mathrm{~s}^{2}\right)^{2} \mathrm{D}$, $(4 \mathrm{p})^{2} \mathrm{P}^{\mathrm{o}}$, and all the pseudostate members listed in Table 3 except for the alkaline-like states $(5 s)^{2} \mathrm{~S}$ and $(5 \mathrm{p})^{2} \mathrm{P}^{\circ}$. Fine structure was not taken into account in their calculation. Note the rich structure in the energy range below $10 \mathrm{eV}$ or so. In using these results, we shifted the energy scales so that the thresholds agreed with the energies of Tables 1 and 3. Cross sections involving individual $\mathrm{J}$ multiplets were inferred by detailed balancing considerations. In addition, for excitation from the ground state to the two $\left(4 \mathrm{~s}^{2}\right) 2^{\mathrm{D}}$ levels and to the two (4p) ${ }^{2} \mathrm{P}$ levels, the cross sections were multiplied by the energy-dependent correction factor

$$
f(E)=E /\left(E+E_{0}^{e x p}-E_{0}^{c a l}\right),
$$

where $E_{0}^{e x p}$ and $E_{0}^{c a l}$ are the experimental and calculated thresholds, respectively.

Reaction rates $\kappa=\langle\sigma v\rangle$ were constructed by averaging with respect to a Maxwellian distribution, via standard Gauss-Laguerre integration.

Our model also requires excitation rates to the two omitted pseudostate members noted above. The optically allowed transitions are, in L-S coupling,

$$
(4 \mathrm{~s})^{2} \mathrm{~S} \rightarrow(5 \mathrm{p})^{2} \mathrm{P}^{\circ}, \quad\left(4 \mathrm{~s}^{2}\right)^{2} \mathrm{D} \rightarrow(5 \mathrm{p})^{2} \mathrm{P}^{\circ}, \quad(4 \mathrm{p})^{2} \mathrm{P}^{\mathrm{o}} \rightarrow(5 \mathrm{~s})^{2} \mathrm{~S} .
$$

We estimate their rates by employing the Van Regemorter formula [102]:

$$
\kappa_{a b}\left(T_{e}\right)=\frac{16\left(2 \pi^{3}\right)^{1 / 2} a_{0}^{2} E_{0}^{2} f_{a b}}{\left(3 m_{e} T_{e}\right)^{1 / 2} \Delta E} \tilde{g}\left(\Delta E / T_{e}\right) \exp \left(-\Delta E / T_{e}\right),
$$

where $f_{a b}$ is the oscillator strength and $\tilde{g}\left(\Delta E / T_{e}\right)$ is a Maxwellian-averaged effective Gaunt factor $\left(\Delta E\right.$ is the threshold energy and $\left.E_{0}=13.6 \mathrm{eV}\right)$.

The final excitation rates are shown in Fig. 15.

\section{A.3 Ionization}

Like copper excitation cross sections, the ionization cross sections are difficult to measure near threshold. The earliest ground state measurements were reported by Pavlov et al. [103] and by Crawford [104]. The former data began at threshold, while the latter began at about 
$46 \mathrm{eV}$. These were followed a few years later by measurements of Schroeer at al. [105], which began at $40 \mathrm{eV}$ and were systematically higher than the earlier data. The latest and most comprehensive data are due to Freund et al. [106], who obtained results from threshold to $200 \mathrm{eV}$ via a crossed-beam apparatus. These are reasonably close to the data of Pavlov et al. Although the nominal uncertainty was $10 \%$, the authors estimated their $\mathrm{Cu}$ beam to contain about $30 \%$ of its population in the ${ }^{2} \mathrm{D}$ level.

A calculation based on a binary encounter model has been given by Rescigno [107]. This tends to agree with Pavlov et al. and Freund et al. at low energies and with Schroeer et al. at higher energies.

Finally, Lotz [108], has developed an empirical formula according to which electrons are treated as though they could be removed independently from the various subshells. For the ground state, this gives a result quite close to the measurements of Freund et al. For excited states, of course, no comparison is available. On the basis of these considerations, the Lotz formula was considered appropriate for describing ionization in the present model.

The Lotz cross section has the form

$$
\sigma(E)=\sum_{i} \eta\left(E-P_{i}\right) a_{i} n_{i} \frac{\ln \left(E / P_{i}\right)}{E P_{i}}\left\{1-b_{i} \exp \left[-c_{i}\left(E / P_{i}-1\right)\right]\right\},
$$

where $i$ runs over subshells. Each subshell is characterized by $n_{i}$ electrons of binding energy $P_{i}$, and by the empirical constants $a_{i}, b_{i}$, and $c_{i}$. The step function $\eta\left(E-P_{i}\right)$ ensures proper behavior at threshold. The general results are expected to be valid within 30 or 40 percent at the maximum of the cross section.

Table 5 displays the reactions pertinent to the states in our model, and Table 6 lists the appropriate parameters. For ionization of the ground state, for example, there are two terms in the sum, one corresponding to removal of a $4 \mathrm{~s}$ electron and the other corresponding to removal of a $3 \mathrm{~d}^{10}$ electron. In these terms, we use parameters appropriate to the $4 \mathrm{~s}$ subshell and $3 \mathrm{~d}^{10}$ subshell, respectively. (Deeper subshells are unimportant in the energy range of interest.) Note that the formulas for the six pseudostate elements with core $4 \mathrm{p}^{\prime}$ (fourth level of Table 5) are similar except for differences in the binding energies (ion energy minus initial energy). The Lotz cross sections and the data of Freund et al. are shown in Fig. 16.

The ionization rates, also shown in Fig. 16, were calculated in the same fashion as the excitation rates.

\section{A.4 Momentum Transfer}

The electron-atom momentum transfer cross section is derived from the elastic differential cross section by insertion of a factor of $1-\cos \theta$ before the integration over angles. It is needed for calculating the plasma resistivity and the electron-atom energy exchange rate. Scheibner and Hazi [99] give this cross section for the ground state.

The sole measurement of this quantity in $\mathrm{Cu}$ appears to have been performed by Trajmar and coworkers [92, 93] (again the second reference supersedes the first). The authors give five energy points for scattering from the ground state and four points for the states $\left(4 \mathrm{~s}^{2}\right)^{2} \mathrm{D}_{5 / 2}$, $\left(4 \mathrm{~s}^{2}\right)^{2} \mathrm{D}_{3 / 2}$, and $(4 \mathrm{~s})^{2} \mathrm{P}$, with the lowest point at $6 \mathrm{eV}$. The ground state cross section has 
Table 5: Ionization Reactions for Lotz Cross Section

\begin{tabular}{|c|c|c|c|}
\hline State(s) & \multicolumn{3}{|c|}{$\begin{array}{l}\text { Reactions } \\
\end{array}$} \\
\hline$(4 \mathrm{~s})^{2} \mathrm{~S}$ & $\begin{array}{c}3 \mathrm{~d}^{10} 4 \mathrm{~s} \\
m\end{array}$ & $\vec{\rightarrow}$ & $\begin{array}{l}3 \mathrm{~d}^{10}+\mathrm{e} \\
3 \mathrm{~d}^{9} 4 \mathrm{~s}+\mathrm{e}\end{array}$ \\
\hline$\left(4 s^{2}\right)^{2} D$ & $\begin{array}{c}3 \mathrm{~d}^{9} 4 \mathrm{~s}^{2} \\
\pi\end{array}$ & $\vec{\rightarrow}$ & $\begin{array}{l}3 d^{10}+e \\
3 d^{8} 4 s^{2}+e\end{array}$ \\
\hline$(4 p)^{2} \mathrm{P}^{\circ}$ & $3 \mathrm{~d}^{10} 4 \mathrm{p}$ & $\begin{array}{l}\vec{\rightarrow} \\
\quad\end{array}$ & $\begin{array}{l}3 d^{10}+e \\
3 d^{9} 4 p+e\end{array}$ \\
\hline $\begin{array}{l}\left(4 \mathrm{p}^{\prime}\right)^{4} \mathrm{P}^{\circ},\left(4 \mathrm{p}^{\prime}\right)^{4} \mathrm{~F}^{\circ},\left(4 \mathrm{p}^{\prime}\right)^{4} \mathrm{D}^{\circ} \\
\left(4 \mathrm{p}^{\prime}\right)^{2} \mathrm{~F}^{\mathrm{o}},\left(4 \mathrm{p}^{\prime}\right)^{2} \mathrm{P}^{\circ},\left(4 \mathrm{p}^{\prime}\right)^{2} \mathrm{D}^{\circ}\end{array}$ & $\begin{array}{c}3 \mathrm{~d}^{9} 4 \mathrm{~s} 4 \mathrm{p} \\
\eta \\
\eta\end{array}$ & $\begin{array}{l}\vec{\rightarrow} \\
\vec{\rightarrow} \\
\rightarrow\end{array}$ & $\begin{array}{l}3 d^{10}+e \\
3 d^{9} 4 s+e \\
3 d^{9} 4 p+e \\
3 d^{8} 4 s 4 p+e\end{array}$ \\
\hline$(5 s)^{2} S$ & $\begin{array}{c}3 \mathrm{~d}^{10} 5 \mathrm{~s} \\
\eta\end{array}$ & $\vec{\rightarrow}$ & $\begin{array}{l}3 \mathrm{~d}^{10}+\mathrm{e} \\
3 \mathrm{~d}^{9} 5 \mathrm{~s}+\mathrm{e}\end{array}$ \\
\hline$(5 p)^{2} \mathrm{P}^{\circ}$ & $\begin{array}{c}3 \mathrm{~d}^{10} 5 \mathrm{p} \\
"\end{array}$ & $\vec{\rightarrow}$ & $\begin{array}{l}3 d^{10}+e \\
3 d^{9} 5 p+e\end{array}$ \\
\hline$(4 d)^{2} D$ & $\begin{array}{c}3 \mathrm{~d}^{10} 4 \mathrm{~d} \\
"\end{array}$ & $\vec{\rightarrow}$ & $\begin{array}{l}3 d^{10}+e \\
3 d^{9} 4 d+e\end{array}$ \\
\hline
\end{tabular}

Table 6: Parameters for Lotz Cross Section

\begin{tabular}{|c|c|c|c|c|c|c|}
\hline Subshell & n & a & b & c & Ion Configuration & Energy \\
\hline $3 \mathrm{~d}^{9}$ & $\overline{9}$ & 1.6 & 0.95 & 0.14 & $3 d^{10}$ & 7.724 \\
\hline $3 d^{10}$ & 10 & 1.4 & 0.96 & 0.13 & $3 d^{9} 4 s$ & 10.4 \\
\hline $4 \mathrm{~s}$ & 1 & 4.0 & 0. & 0. & $3 d^{9} 4 p$ & 16.0 \\
\hline $4 s^{2}$ & 2 & 4.0 & 0. & 0. & $3 d^{8} 4 s^{2}$ & 16.4 \\
\hline $4 p$ & 1 & 4.0 & 0. & 0. & $3 d^{8} 4 s 4 p$ & 21.1 \\
\hline $4 \mathrm{~d}$ & 1 & 4.0 & 0.30 & 0.60 & $3 d^{9} 5 s$ & 21.1 \\
\hline $5 \mathrm{~s}$ & 1 & 4.0 & 0. & 0. & $3 d^{9} 4 d$ & 21.9 \\
\hline $5 p$ & 1 & 4.0 & 0. & 0. & $3 d^{9} 5 p$ & 22.6 \\
\hline
\end{tabular}


about the same shape as the Scheibner-Hazi calculation (some structure at low energy and an exponential falloff at higher energies), but it is systematically higher by about a factor of about six. This may not be significant, since the overall normalization of the data is subject to dispute. The (4p) ${ }^{2} \mathrm{P}$ cross section is about $1 / 6$ the of the ground state cross section, and the cross sections from the $\left(4 \mathrm{~s}^{2}\right)^{2} \mathrm{D}$ levels are lower by another factor of six. All the curves are plotted in Fig. 17. Note that the ground state cross section starts at about $10^{-14} \mathrm{~cm}^{2}$ at low energy, rises to a peak at a fraction of an $\mathrm{eV}$, and thereafter falls off approximately as $1 / E$. As expected, it shows some structure between 2 and $10 \mathrm{eV}$.

In the model, we employ the Scheibner-Hazi result for the ground state cross section, and we assume that the cross sections from other levels scale as observed experimentally. The pseudostate cross section is taken to be the same as that for $(4 \mathrm{p})^{2} \mathrm{P}$. The curves are plotted in Fig. 17.

\section{A.5 Recombination}

Because of the existence of autoionizing states, recombination is expected to be an extremely complicated process in the copper atom. Lacking experimental information, however, we simply assume that three-body recombination proceeds as in the hydrogen atom, where classical Monte Carlo calculations [80] are in reasonable agreement with experiment. In this treatment, the recombination rate coefficient has the form

$$
\kappa_{\text {rec }}=\gamma a^{5}\left(2 T_{e} / m_{e}\right)^{1 / 2},
$$

with $a=e^{2} / 4 \pi \epsilon_{0} T_{e}$ and $\gamma \simeq 0.54$. Note that this varies at low temperature as $T_{e}^{-9 / 2}$. In conditions characteristic of the laser afterglow $\left(T_{e} \simeq 0.2 \mathrm{eV}, n_{e} \simeq 5 \times 10^{13} \mathrm{~cm}^{-3}\right)$, the time scale is about $140 \mu \mathrm{s}$. Thus recombination acts as a bottleneck in the kinetics.

\section{B Neon Rates}

The excitation cross section from the Ne ground state to the $3 \mathrm{~s}$ state is taken from a calculation by Theodosiou [109]. The excitation cross section from ground to the 3p state is taken from experiment [110]. The ground state ionization cross section is taken from a measurement by Fletcher and Cowling [111]. The 3s ionization cross section is taken from experiment [112], while the $3 p$ ionization is taken from a calculation by Hyman [113]. Note that the radiation rate from $3 \mathrm{~s}$ to ground $\left(1.78 \times 10^{8} \mathrm{~s}^{-1}\right)$ requires a trapping correction.

The final excitation and ionization rate coefficients are plotted in Fig. 18.

The momentum transfer cross section has been measured in several experiments. We employ values recommended by Itikawa [114], which are taken from measurements by Robertson [115] and Sol et al. [116]. The cross section and the resulting rates are shown in Fig. 19. Note that the cross section is more than an order of magnitude less than that for copper. Electron-Ne collisions dominate, however, since the neon density typically exceeds the copper density by two orders of magnitude. 


\section{Hydrogen Rates}

Electron-impact excitation and ionization rates of the hydrogen atom were taken from Vriens and Smeets [117]. The cross section for electron detachment from the negative ion was taken from values recommended by Massey [118]. The attachment rate was calculated via detailed balance, i.e.

$$
\kappa\left(2 \mathrm{e}+\mathrm{H} \rightarrow \mathrm{e}+\mathrm{H}^{-}\right)=\kappa\left(\mathrm{e}+\mathrm{H}^{-} \rightarrow 2 \mathrm{e}+\mathrm{H}\right)\left(\frac{h^{2}}{2 \pi m_{e} k T_{e}}\right)^{3 / 2} \exp \left(E_{H^{-}} / k T_{e}\right),
$$

with binding energy $E_{H^{-}}=0.76 \mathrm{eV}$. In comparison, the radiative attachment rate [119] is insignificant. The e-H momentum transfer cross section was taken from a curve recommended by Itakawa [120].

For dissociation of the hydrogen molecule, we used the main channel, e $+\mathrm{H}_{2} \rightarrow \mathbf{e}+$ $\mathrm{H}(1 \mathrm{~s})+\mathrm{H}(1 \mathrm{~s})$, with a rate recommended by Janev et al. [121]. The inverse rate was taken from detailed balance,

$$
\kappa\left(\mathrm{e}+\mathrm{H}+\mathrm{H} \rightarrow \mathrm{e}+\mathrm{H}_{2}\right)=\kappa\left(\mathrm{e}+\mathrm{H}_{2} \rightarrow \mathrm{e}+\mathrm{H}+\mathrm{H}\right)\left(\frac{h^{2}}{\pi m_{H} k T_{e}}\right)^{3 / 2} Q_{r o t} Q_{v i b} \exp \left(E_{H_{2}} / k T_{e}\right)
$$

with $E_{H_{2}}=4.48 \mathrm{eV}$ the molecular binding energy. The rotational and vibrational partition functions have the form

$$
Q_{\text {rot }}(T)=T / \Theta_{r}, \quad Q_{v i b}(T)=\left[1-\exp \left(-\Theta_{v} / T\right)\right]^{-1},
$$

with $\Theta_{r}=2 \mathrm{~K}$ and $\Theta_{v}=2000 \mathrm{~K}$. The e- $\mathrm{H}_{2}$ momentum transfer rate was taken from Itikawa [114].

These rates are plotted in Figures 20, 21, and 22.

\section{Radiation Trapping}

After an excited atom in a vapor spontaneously decays, the emitted photon may travel only a certain distance before being absorbed by another atom in the lower state. This distance depends not only on the density of lower state atoms but also on the frequency of the photon, which is distributed within the atomic emission spectrum. After a spontaneous lifetime, the absorbing atom will emit another photon, which will travel another frequencydependent mean free path before again being absorbed. Eventually a photon in this chain will escape from the system. This process of successive emission and absorption, called radiative trapping, increases the effective lifetime of the transition. To describe it in detail, via the radiation transport equation, is far beyond the scope of most discharge models. Instead, following Holstein $[122,123]$, we adopt a global approach in which the effective decay rate is reduced from the spontaneous rate by an escape factor. In the case of the copper resonance lines, for example, the effective rate is decreased by a factor of approximately 500 , depending 
on conditions. This correction plays a critical role in the model, since, in its absence, an inversion could not even be attained.

The escape factor depends on the geometry of the system and on the emission lineshape. Although most photons are emitted near line center, where lines are usually Gaussian (due to Doppler broadening), they are quickly absorbed. The relatively fewer photons emitted far from line center, where lines tend to be Lorentzian (due to pressure broadening), are responsible for the bulk of the radiative energy transport. Following Holstein, we calculate the escape factor separately for the two kinds of broadening. We then make the ansatz that the effective escape factor is the greater of these. For the resonance lines, the dominant mechanism is always pressure broadening, while for other lines the dominant mechanism may change during the pulse. Although this treatment obviously represents an idealization, it seems fairly reasonable. In any case, not much more can easily be done.

In calculating the escape factor, we employ the Holstein treatment for cylindrical geometry, except that we integrate over an arbitrary radial density profile rather than solving for the slowest mode.

An important parameter in this treatment is the absorption at line center, given by

$$
k=\frac{\lambda^{2} n_{1} A g_{2}}{4 \pi^{2} g_{1} \Delta \nu}
$$

where the indices 1 and 2 refer to the lower state and upper state, respectively, and $\Delta \nu$ is the linewidth. For pressure broadening the linewidth can be parametrized as $\Delta \nu=n_{0} \sigma v_{r} / \pi$, with $n_{0}$ the density of buffer gas atoms, $\sigma$ an approximate cross section, and $v_{r}$ the relative speed. For Doppler broadening, the appropriate linewidth is

$$
\Delta \nu=2 v_{t h} / \sqrt{\pi} \lambda,
$$

with $v_{t h}=(2 k T / m)^{1 / 2}$ the thermal speed.

In the special case of insufficient absorption at line center, trapping can clearly be ignored. We parametrize this situation by the condition $k r_{\text {eff }}<1$, with $r_{\text {eff }}$ an effective distance determined by the geometry of the system. In a cylinder, it seems reasonable to set $r_{\text {eff }}$ equal to the radius. In the case of a septum, we estimate $r_{\text {eff }}=a / 2$.

After a calculation, we find that the escape factor is given an expression of the form

$$
f=\frac{\alpha c_{m} b_{m}}{(k a)^{m}}
$$

where

$$
\alpha= \begin{cases}\pi^{-1 / 2} & \text { pressure broadening, } \\ {\left[\pi \ln \left(k r_{e f f}\right)\right]^{-1 / 2}} & \text { Doppler broadening, }\end{cases}
$$

and $m$ is $1 / 2$ and 1 in the respective cases. These last two parameters are such that the transmission function is $T(r)=\alpha(k r)^{-m}$. Also $c_{m}$ is the constant

$$
c_{m}=\frac{m^{2} \Gamma(m)}{(m+1) 2^{m}[\Gamma(m / 2+1 / 2)]^{2}},
$$


which reduces to 0.139 and $1 / 4$ in the respective cases. Finally, $b_{m}$ represents an integral over the radial profile:

$$
b_{m}=\int_{0}^{1} d u u \bar{n}(u) h_{m}(u), \quad(u=r / a),
$$

where $\bar{n}(u)=n(r) /<n>$ is the profile factor, and $h_{m}$ is the function

$$
h_{m}(u)=\frac{2}{m} \int_{0}^{\pi} d \theta \frac{1-u \cos \theta}{\left(1+u^{2}-2 u \cos \theta\right)^{1+m / 2}} .
$$




\section{References}

[1] W. T. Walter, N. Solimene, M. Piltch, and G. Gould, "Efficient pulsed gas discharge lasers," IEEE J. Quantum Electron. QE-2, 474-479 (1966).

[2] B. E. Warner, C. D. Boley, J. J. Chang, E. P. Dragon, M. A. Havstad, M. Martinez, and W. McLean II, "Industrial applications of high-power copper vapor lasers," NATO Advanced Research Workshop on Pulsed Metal Vapour Lasers, St. Andrews, Scotland, UK, August, 1995.

[3] J. J. Chang, B. E. Warner, C. D. Boley, and E. P. Dragon, "High-power copper vapour lasers and applications," NATO Advanced Research Workshop on Pulsed Metal Vapour Lasers, St. Andrews, Scotland, UK, August, 1995.

[4] J. L. Miller and T. Kan, "Metastable decay rates in a Cu-metal-vapor laser," J. Appl. Phys. 50, 3849-3851 (1979).

[5] R. S. Hargrove, R. Grove, and T. Kan, "Copper vapor laser unstable resonator oscillator and oscillator-amplifier characteristics," IEEE J. Quantum Electron. QE-15, 12281233 (1979).

[6] M. J. Kushner, "A self-consistent model for high repetition rate copper vapor lasers," IEEE J. Quantum Electron. QE-17, 1555-1565 (1981).

[7] M. J. Kushner and B. E. Warner, "Large-bore copper-vapor lasers: kinetics and scaling issues,” J. Appl. Phys. 54, 2970 (1983).

[8] W. A. Molander, "Measurement of ground state copper density using hook spectroscopy," Metal Vapor, Deep Blue, and Ultraviolet Lasers, SPIE Vol. 1041, pp. 11-18, 1989.

[9] J. J. Chang, C. D. Boley, M. W. Martinez, W. A. Molander, and B. E. Warner, "Beam characteristics of a large-bore copper laser with a radiatively cooled plasma," Gas, Metal Vapor, and Free-Electron Lasers and Applications, SPIE Vol. 2118, pp. 2-8, 1994.

[10] J. J. Chang, "Copper-laser oscillator with adjoint-coupled self-filtering injection," Opt. Lett. 20, 575-577 (1995).

[11] G. G. Petrash, "Pulsed gas-discharge lasers," Sov. Phys. Usp. 14, 747-765 (1972).

[12] A. A. Isaev, M. A. Kazaryan, and G. G. Petrash, "Possibility of generation of high average laser powers in the visible part of the spectrum," Sov. J. Quantum Electron. 3, 521-523 (1974).

[13] P. A. Bokan and V. I. Solomonov, "Mechanism of laser action in copper vapor," Sov. J. Quantum Electron. 3, 481-483 (1974). 
[14] A. A. Isaev, M. A. Kazaryan, G. G. Petrash, S. G. Rautian, and A. M. Shalagin, "Shaping of the output beam in a pulsed gas laser with an unstable resonator," Sov. J. Quantum Electron. 7, 746-752 (1977).

[15] V. A. Burmakin, A. N. Evtyunin, M. A. Lesnoĭ, and V. I. Bylkin, "Long-life sealed copper vapor laser," Sov. J. Quantum Electron. 8, 574-576 (1978).

[16] P. A. Bokhan, V. A. Gerasimov, V. I. Solomonov, and V. B. Shcheglov, "Stimulated emission mechanism of a copper vapor laser," Sov. J. Quantum Electron. 8, 1220-1227 (1978).

[17] V. M. Batenin, I. I. Klimovskii, A. V. Morozov, and L. A. Selezneva, "Spectral composition of induced emission from a copper vapor laser and its time evolution," High Temperature, Vol. 17, pp. 406-411, Consultants Bureau, NY, 1979.

[18] A. A. Isaev, "Spectral composition of stimulated radiation from a pulsed copper vapor laser," Sov. J. Quantum Electron. 10, 336-341 (1980).

[19] V. M. Batenin, I. I. Klimovskii, M. A. Lesnoï, and L. A. Selezneva, "Plasma parameters in the afterglow of a copper vapor laser," Sov. J. Quantum Electron. 10, 563-566 (1980).

[20] P. A. Bokhan, V. I. Silant'ev, and V. I. Solomonov, "Mechanism for limiting the repetition frequency of pulses from a copper vapor laser," Sov. J. Quantum Electron. 10, 724-727 (1980).

[21] S. V. Arlantsev, V. V. Buchanov, L. A. Vasil'ev, É. I. Molodykh, V. V. Tykotskii, and V. I. Yurchenko, "Numerical investigation of a pulse-periodic copper vapor laser," Sov. J. Quantum Electron. 10, 1350-1354 (1980).

[22] A. Ya. Litvinenko, V. I. Kravchenko, and A. N. Egorov, "Measurement of the lifetimes of the lower active levels of a copper vapor laser," Sov. J. Quantum Electron. 13, 778-781 (1983).

[23] V. V. Zubov, N. A. Lyabin, V. I. Mishin, M. L. Muchnik, G. D. Parshin, E. Ya. Chernyak, and A. D. Chursin, "Investigation of a copper vapor laser with a long service life and improved excitation pulse parameters," Sov. J. Quantum Electron. 13, 1266-1268 (1983).

[24] B. L. Borovich, V. V. Buchanov, and É. I. Molodykh, "Numerical modeling of an electron-beam-pumped copper vapor laser," Sov. J. Quantum Electron. 14, 680-684 (1984).

[25] B. L. Borovich and N. I. Yurchenko, "Analysis of the excitation and relaxation kinetics in a copper vapor laser excited by a longitudinal discharge," Sov. J. Quantum Electron. 14, 1391-1400 (1984). 
[26] A. A. Isaev, G. G. Petrash, and I. V. Ponomarev, "Relaxation of metastable atoms during the afterglow in a copper vapor laser," Sov. J. Quantum Electron. 16, 1512-1516 (1986).

[27] A. A. Isaev, V. V. Kazakov, M. A. Lesnoì, S. V. Markova, and G. G. Petrash, "Decay of metastable states and its influence on the lasing characteristics of a copper vapor laser," Sov. J. Quantum Electron. 16, 1517-1522 (1986).

[28] A. A. Isaev, V. T. Mikhkel'soo, G. G. Petrash, V. É. Peét, I. V. Ponomarev, and A. B. Treshchalov, "Kinetics of excitation of the active levels of a copper vapor laser by pulse pairs," Sov. J. Quantum Electron. 18, 1577-1579 (1988).

[29] I. P. Zapesochnyi, V. A. Kel'man, I. I. Klimovskii, L. A. Selezneva, and V. Yu. Fuchko, "Nonuniformity of a discharge in a copper-vapor laser and its effect on gas temperature," High Temperature, Vol. 26, pp. 505-514, Consultants Bureau, NY, 1989.

[30] A. A. Isaev, V. T. Mikhkel'soo, G. G. Petrash, V. É. Peét, I. V. Ponomarev, A. B. Treshchalov, and N. I. Yurchenko, "Spatial-temporal kinetics of the ground state population of the copper atom in a copper vapor laser," Sov. J. Quantum Electron. 19, 759-765 (1989).

[31] A. A. Isaev, V. T. Mikhkel'soo, G. G. Petrash, V. É. Peét, I. V. Ponomarev, and A. B. Treshchalov, "Atomic levels population and depopulation kinetics in Cu-vapor laser," Metal Vapor, Deep Blue, and Ultraviolet Lasers, SPIE Vol. 1041, pp. 40-46, 1989.

[32] N. A. Lyabin, V. V. Zubov, and A. D. Chursin, "Active element utilizing copper vapor for high-power master-oscillator-amplifier laser systems," Sov. J. Quantum Electron. 20, 20-23 (1990).

[33] G. G. Petrash, "Basic physical processes in pulsed metal vapor lasers," High-Power Gas Lasers, SPIE Vol. 1225, pp. 216-227, 1990.

[34] I. I. Klimovskii, "Making a repetitively pulsed copper-vapor laser with a specific power of over $1 \mathrm{~W} / \mathrm{cm}^{3}$ and a practical efficiency at the $8 \%$ level," High Temperature, Vol. 27, pp. 952-959, Consultants Bureau, NY, 1990.

[35] R. I. Okunev, L. N. Pakhomov, and A. G. Petrakov, "Selection of the emission spectrum of a copper vapor laser," Sov. Tech. Phys. Letter. 17, 449-451 (1991).

[36] M. Nikonchuk and I. Polyakov, "Focal plane intensity distribution of copper vapor laser with different unstable resonators," Gas and Metal Vapor Lasers and Applications, SPIE Vol. 1412, pp. 72-78, 1991.

[37] S. M. Gladkov, A. M. Zheltikov, O. S. Il'yasov, A. A. Isaev, and N. I. Koroteev, "CARS diagnostics of the active medium of a metal vapor laser," Sov. J. Quantum Electron. 21, 659-663 (1991). 
[38] B. B. Buchanov, M. A. Kazaryan, É. I. Molodykh, and V. A. Shcheglov, "Feasibility of constructing a cw copper vapor-flow laser," Sov. J. Quantum Electron. 24, 959-962 (1994).

[39] P. A. Bokhan and E. I. Molodyka, "Output limiting mechanisms and the prospects for metal vapour lasers with average power above $1 \mathrm{~kW} / \mathrm{m}$," NATO Advanced Research Workshop on Pulsed Metal Vapour Lasers, St. Andrews, Scotland, UK, August, 1995.

[40] M. A. Kazaryan, NATO Advanced Research Workshop on Pulsed Metal Vapour Lasers, St. Andrews, Scotland, UK, August, 1995.

[41] I. Smilanski, A. Kerman, L. A. Levin, and G. Erez, "Scaling of the discharge heated copper vapor laser," Optics Comm. 25, 79-82 (1978).

[42] I. Smilanski, "Copper hooks - investigation of the copper-vapor-laser kinetics," Proc. International Conf. Lasers '79, Orlando, FL, pp. 327-334, 1979.

[43] I. Smilanski, G. Erez, A. Kerman, and L. A. Levin, "High-power, high-pressure, discharge-heated copper vapor laser," Optics Comm. 30, 70-74 (1979).

[44] J. Tenenbaum, I. Smilanski, S. Lavi, L. A. Levin, and G. Erez, "Kinetic investigation of the upper laser levels of the copper vapor laser," Optics Comm. 36, 391-394 (1981).

[45] M. Amit, S. Lavi, G. Erez, and E. Miron, "Temporal and spatial properties of an oscillator-amplifier copper vapor laser," Optics Commun. 62, 110-114 (1987).

[46] I. Smilanski, S. Gabay, and Z. Karni, "Ground state depletion in copper vapor lasers," Metal Vapor, Deep Blue, and Ultraviolet Lasers, SPIE Vol. 1041, pp. 34-39, 1989.

[47] M. Lando, D. Belker, A. Lerrer, H. Lotem, A. Dikman, G. Bialolanker, S. Levi, and S. Gabay, "Modified off-axis unstable resonator for copper vapor laser," Gas and Metal Vapor Lasers and Applications, SPIE Vol. 1412, pp. 19-26, 1991.

[48] C. Yamanaka, E. Fujiwara, T. Naka, T. Takeda, T. Yamanaka, and C. Yamanaka, "Copper vapor lasers for isotope separation" Technology Reports of Osaka Univ. 36, 353-359 (1986).

[49] Y. Izawa, T. Shimotsu, C. Yamanaka, N. Nakashima, E. Fujiwara, T. Yamanaka, S. Nakai, S. Kuruma, T. Takeda, Y. Ojima, and C. Yamanaka, "Density measurements of the lower level of a copper vapor laser," Metal Vapor, Deep Blue, and Ultraviolet Lasers, SPIE Vol. 1041, pp. 19-24, 1989.

[50] K. Kuroda, T. Omatsu, T. Shimura, M. Chihara, and I. Ogura, "Parametric study on the second harmonic generation of a copper vapor laser," Metal Vapor, Deep Blue, and Ultraviolet Lasers, SPIE Vol. 1041, pp. 60-66, 1989.

[51] E. Fujiwara, C. Yamanaka, N. Nakashima, H. Deguchi, C. Yamanaka, E. Murata, and T. Hatakeyama, "Copper vapor laser operated by a solid state switch, High-Power Gas Lasers, SPIE Vol. 1225, pp. 240-247, 1990. 
[52] S. Shirayama, T. Mikatsura, H. Ueda, and C. Konagai, "Laser system for isotope separation," High-Power Gas Lasers, SPIE Vol. 1225, pp. 279-288, 1990.

[53] T. Omatsu, K. Kuroda, T. Shimura, M. Chihara, M. Itoh, and I. Ogura, "Non-collinear iteration model of the second harmonic generation of a copper laser model," Optics Commun. 79, 125-130 (1990).

[54] N. Aoki, H. Kimura, C. Konagai, S. Shirayama, T. Miyazawa, and T. Takahashi, "Highpower copper vapor laser development", Gas and Metal Vapor Lasers and Applications, SPIE Vol. 1412, pp. 2-11, 1991.

[55] T. Omatsu, T. Takase, and K. Kuroda, "Evolution of the spatial coherence in a copper vapor laser," Optics Commun. 92, 50-56 (1992).

[56] T. Omatsu, T. Takase, and K. Kuroda, "Saturation of the conversion efficiency of second generation harmonic generation in a copper vapor laser," Optics Commun. 97, 65-68 (1993).

[57] R. R. Lewis, G. Maldonado, and C. E. Webb, "Copper vapor lasers: recent advances," Metal Vapor, Deep Blue, and Ultraviolet Lasers, SPIE Vol. 1041, pp. 54-59, 1989.

[58] A. J. Kearsley, "Copper vapor lasers," High-Power Gas Lasers, SPIE Vol. 1225, pp. 270-278, 1990.

[59] C. E. Webb, "Copper vapour laser pumping of dye lasers in atomic and nuclear research," Inst. Phys. Conf. Ser. No. 114, Section 9, RIS 90, Varese, Italy, 1990.

[60] D. Kapitan, H. Booth, G. J. Murray, G. P. Hogan, and C. E. Webb, Proc. XI International Symp. on Gas Flow and Chemical Lasers and High Power Laser Conf., Edinburgh, UK, 1996.

[61] M. D. Ainsworth, D. W. Coutts, and J. A. Piper, "Wavelength extension of copper vapour lasers," Metal Vapor, Deep Blue, and Ultraviolet Lasers, SPIE Vol. 1041, pp. 92-103, 1989.

[62] D. J. W. Brown, R. Künnemeyer, and A. I. McIntosh, "Radial excited-state density effects in a small-bore copper vapour laser," Metal Vapor, Deep Blue, and Ultraviolet Lasers, SPIE Vol. 1041, pp. 25-33, 1989.

[63] D. J. W. Brown, R. Künnemeyer, and A. I. McIntosh, "Time-resolved measurements of excited state densities in a copper vapor laser," IEEE J. Quantum Electron. 26, 1609-1619 (1990).

[64] D. W. Coutts and J. A. Piper, "1 W average power by second harmonic and sum frequency generation from a single medium scale copper vapor laser," IEEE J. Quantum Electron. QE-28, 1761-1764 (1992). 
[65] R. J. Carman, D. J. W. Brown, and J. A. Piper, "A self-consistent model for the discharge kinetics in a high-repetition rate copper vapor laser," IEEE J. Quantum Electron. 30, 1876-1895 (1994).

766] D. W. Coutts, "Time-resolved beam divergence from a copper vapor laser with unstable resonator," IEEE J. Quantum Electron. 31, 330-342 (1995).

[67] M. J. Withford and D. J. W. Brown, IEEE J. Selected Topics Quantum Electron. 1, 779 (1995).

[68] M. J. Withford, D. J. W. Brown, and J. A. Piper, "Investigation of the evolution of trace impurities from a newly constructed copper vapour laser," J. Phys. D 29, 315-321 (1996).

[69] C. D. Boley, "Computational model of a magnetic modulator for copper lasers," Proc. 9th IEEE International Pulsed Power Conf., Albuquerque, NM, pp. 704-707, 1993.

[70] D. A. Leonard, "A theoretical description of the 5106- $\AA$ pulsed copper vapor laser," IEEE J. Quantum Electron. QE-3, 380-381 (1967).

[71] G. M. Kull, N. Solimene, and W. T. Walter, "Modeling of pulsed gas lasers and comparison with experimental results for the copper vapor laser," Proc. Int. Conf. Lasers, Orlando, FL (1979).

[72] K. G. Harstad, "Computer simulated rate processes in copper vapor lasers," IEEE J. Quantum Electron. QE-16, 550-558 (1980).

[73] C. E. Moore, "Atomic Energy Levels," Vol. II, Circular of the National Bureau of Standards 467 (1952).

[74] C. E. Moore, "Atomic Energy Levels," Vol. I, Circular of the National Bureau of Standards 467 (1949).

[75] D. N. Astadjov, N. V. Sabotinov, and N. K. Vuchkov, "Effect of hydrogen on $\mathrm{CuBr}$ laser power and efficiency," Optics Commun. 56, 279-282 (1985).

[76] D. N. Astadjov, N. K. Vuchkov, and N. V. Sabotinov, "Parametric study of the $\mathrm{CuBr}$ laser with hydrogen additives," IEEE J. Quantum Electron. 24, 1927 (1988).

[77] H. N. Hersh, J. Am. Chem. Soc. 75, 1529 (1953).

[78] J. P. Morris and G. R. Zellars, "Vapor pressure of liquid copper and activities in liquid Fe-Cu alloys," J. Metals 8, 1086 (1956).

[79] A. N. Nesmeyanov, Vapor Pressure of the Chemical Elements, Elsevier Publishing Company, Amsterdam (1963).

[80] P. Mansbach and J. Keck, "Monte Carlo trajectory calculations of atomic excitation and ionization by thermal electrons," Phys. Rev. 181, 275 (1969). 
[81] S. I. Braginskii, "Transport Processes in a Plasma," in Reviews of Plasma Physics (edited by M. A. Leontovich), Vol. I, Consultants Bureau, NY, 1965.

[82] E. G. Cook, D. G. Ball, D. L. Birx, J. D. Branum, S. E. Peluso, M. D. Langford, R. D. Speer, J. S. Sullivan, and P. D. Woods, "High average power magnetic modulator for copper lasers," Proc. 8th IEEE International Pulsed Power Conf., San Diego, CA, pp. 537-542, 1991.

[83] C. D. Boley and M. L. Hodgdon, "Model and simulation of hysteresis in magnetic cores," IEEE Trans. Magnetics 25, 3922-3924 (1989).

[84] C. D. Boley, "Simulations of a magnetic pulse compressor," Proc. 8th IEEE International Pulsed Power Conf., San Diego, CA, pp. 704-707, 1991.

[85] A. C. Hindmarsh, "ODEPACK, a systematized collection of ODE solvers," in Scientific Computing, R. S. Stepleman et al. (eds.), North-Holland, Amsterdam, 1983 (Vol. I of IMACS Transactions on Scientific Computing), pp. 55-64.

[86] P. N. Brown and A. C. Hindmarsh, "Reduced storage matrix methods in stiff ODE systems," J. Appl. Math. Comp. 31, 40-91 (1989).

[87] K. L. Hiebert and L. F. Shampine, "Implicitly defined output points for solutions of ODEs," Sandia National Laboratories, SAND80-0180, February, 1980.

[88] A. T. Anderson, Lawrence Livermore National Laboratory, private communication.

[89] J. J. Chang, Lawrence Livermore National Laboratory, private communication.

[90] C. D. Boley and B. E. Warner, "Model of pulse extraction from a copper laser amplifier," Lawrence Livermore National Laboratory, 1997.

[91] A. Bielski, "A critical survey of atomic transition probabilities for CuI," J. Quant. Spectrosc. Radiat. Transfer 15, 463 (1974).

[92] W. Williams and S. Trajmar, "Elastic and inelastic electron scattering at 20 and 60 eV from atomic copper," Phys. Rev. Letters 33, 197 (1974).

[93] S. Trajmar, W. Williams, and S. K. Srivastava, "Electron-impact cross sections for $\mathrm{Cu}$ atoms," J. Phys. B 10, 3323 (1977).

[94] A. Z. Msezane and R. J. W. Henry, "Generalized oscillator strengths for $4 s^{2} S \rightarrow 4 p^{2} P$ transition in $\mathrm{Cu}$ : comparison of theory and experiment," Phys. Rev. Letters 55, 2277 (1985).

[95] L. S. Aleksakhin, A. A. Borovik, V. P. Starodub, and I. I. Shafranjosh, Zh. Prik. Spektrosk. 30, 236 (1979).

[96] P. J. O. Teubner, “Electron scattering from copper," Aus. J. Phys. 44, 259 (1991). 
[97] A. Z. Msezane and R. J. W. Henry, "Electron-impact excitation of atomic copper," Phys. Rev. A 33, 1631 (1986).

[98] K. F. Scheibner, A. U. Hazi, and R. J. W. Henry, "Electron-impact excitation cross sections for transitions in atomic copper," Phys. Rev. A 35, 4869 (1987).

[99] K. F. Scheibner and A. U. Hazi, unpublished.

[100] K. F. Scheibner and A. U. Hazi, "Photodetachment cross section of $\mathrm{Cu}^{-}$," Phys. Rev. A 38, 539 (1988).

[101] C. Flynn, Z. Wei, and B. Stumpf, "Excitation of the copper resonance lines by lowenergy electrons," Phys. Rev. A 48, 1239 (1993).

[102] H. Van Regemorter, "Rate of collisional excitation in stellar atmospheres," Astrophys. J. 136, 906 (1962).

[103] S. I. Pavlov, V. I. Rakhovskii, and G. M. Fedorova, "Measurement of cross sections for ionization by electron impact at low vapor pressures," Sov. Phys. JETP 25, 12 (1967).

[104] C. K. Crawford, "Electron impact ionization cross sections," Tech. Rept. No. 1, Particle Optics Laboratory, Massachusetts Institute of Technology, Cambridge, MA (1967). AFML-TR-67-376.

[105] J. M. Schroeer, D. H. Gündüz, and S. Livingston, "Electron impact ionization cross sections of $\mathrm{Cu}$ and $\mathrm{Au}$ between 40 and $250 \mathrm{eV}$, and the velocity of evaporated atoms," J. Chem. Phys. 58, 5135 (1973).

[106] R. S. Freund, R. C. Wetzel, R. A. Shul, and T. R.Hayes, "Cross-section measurements for electron-impact ionization of atoms," Phys. Rev. A 41, 3575 (1990).

[107] T. N. Rescigno, "Cross sections for electron-impact ionization of copper," Lawrence Livermore National Laboratory, TAMP 81-0101 (1981).

[108] W. Lotz, "Electron-impact ionization cross sections for atoms up to $Z=108$," Z. Physik 232, 101 (1970), and earlier references.

[109] C. E. Theodosiou, "Excitation of neon by impact of slow and heavy projectiles," J. Phys. B 13, L113 (1980).

[110] F. A. Sharpton, R. M. St. John, C. C. Lin, and F. F. Fajen, "Experimental and theoretical studies of electron-impact excitation of neon," Phys. Rev. A 2, 1305 (1970).

[111] J. Fletcher and I. R. Cowling, "Electron impact ionization of neon and argon," J. Phys. B 6, L258 (1973).

[112] D. Ton-That and M. R. Flannery, "Cross sections for ionization of metastable raregas atoms $\left(\mathrm{Ne}^{*}, \mathrm{Ar}^{*}, \mathrm{Kr}^{*}\right.$, and $\left.\mathrm{Xe}^{*}\right)$ and of metastable $\mathrm{N}_{2}^{*}, \mathrm{CO}^{*}$ molecules by electron impact," Phys. Rev. A 15, 517 (1977). 
[113] H. A. Hyman, "Electron-impact Ionization cross sections for excited states of the rare gases ( $\mathrm{Ne}, \mathrm{Ar}, \mathrm{Kr}, \mathrm{Xe}$ ), cadmium, and mercury," Phys. Rev. A 20, 855 (1979).

[114] Y. Itikawa, "Momentum-transfer cross sections for electron collisions with atoms and electrons," Atomic Data and Nuclear Data Tables 21, 69 (1978).

[115] A. G. Robertson, "The momentum transfer cross section for low energy electrons in neon," J. Phys. B 5, 648 (1972).

[116] C. Sol, F. Devos, and J.-C. Gauthier, "Elastic electron-neutral interaction measurements in neon at ultralow energies," Phys. Rev. A 12, 502 (1975).

[117] L. Vriens and A. H. M. Smeets, "Cross-section and rate formulas for electron-impact ionization, excitation, deexcitation, and total depopulation of excited atoms," Phys. Rev. A 22, 940 (1980).

[118] H. S. W. Massey, Negative Ions, Cambridge University Press, 3rd Edition (1976).

[119] H. S. W. Massey, E. H. S. Burhop, and H. B. Gilbody, Electronic and Ionic Impact Phenomena, Vol. II, Oxford Univ. Press, 1969.

[120] Y. Itikawa, "Momentum-transfer cross sections for electron collisions with atoms and molecules," Atomic Data and Nuclear Data Tables 14, 1 (1974).

[121] R. K. Janev, W. D. Langer, K. Evans, Jr., and D. E. Post, Jr., Elementary Processes in Hydrogen-Helium Plasmas, Springer-Verlag, Berlin, 1987.

[122] T. Holstein, "Imprisonment of resonance radiation in gases," Phys. Rev. 72, 1212 (1947).

[123] T. Holstein, "Imprisonment of resonance radiation in gases. II," Phys. Rev. 83, 1159 (1951). 

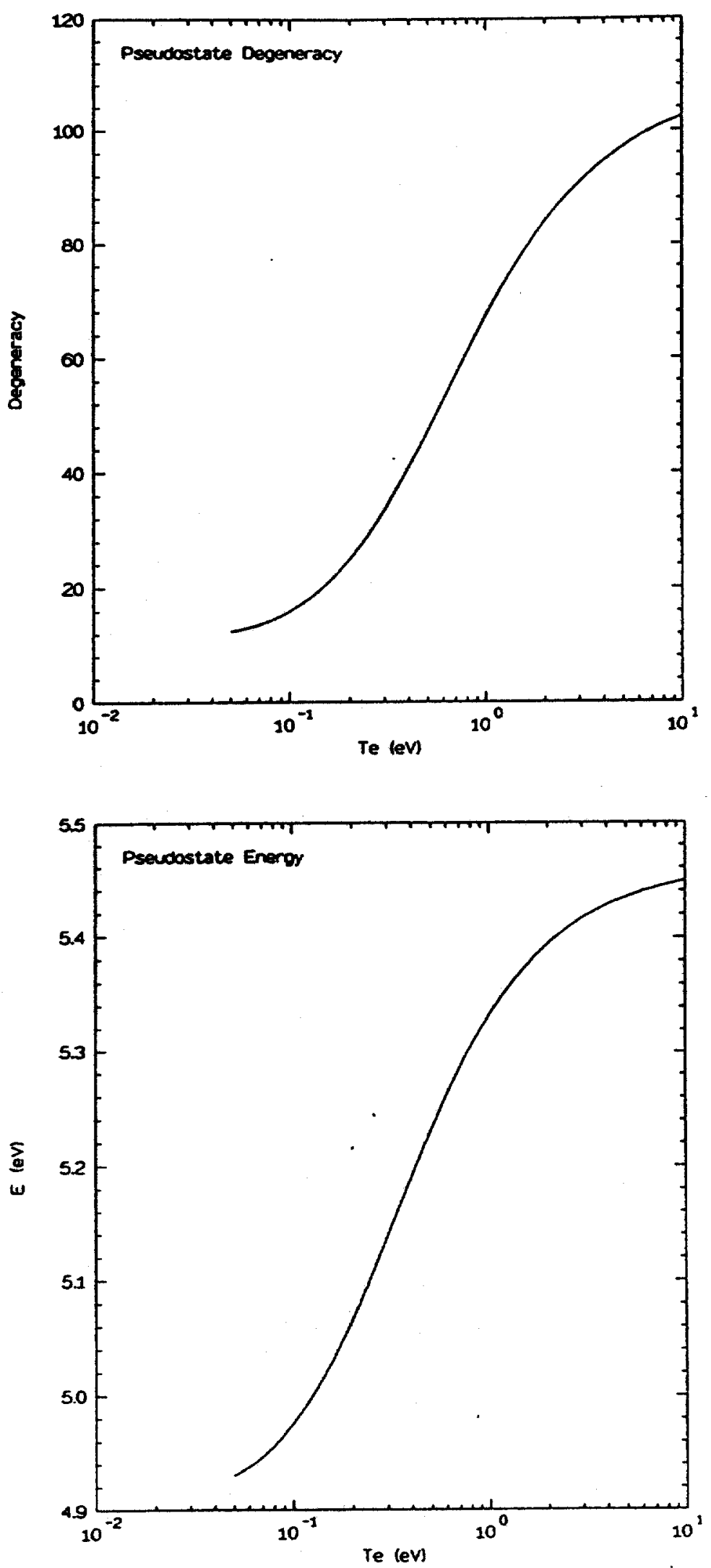

Figure 1: Effective degeneracy of Cu pseudostate; effective energy of the pseudostate. 


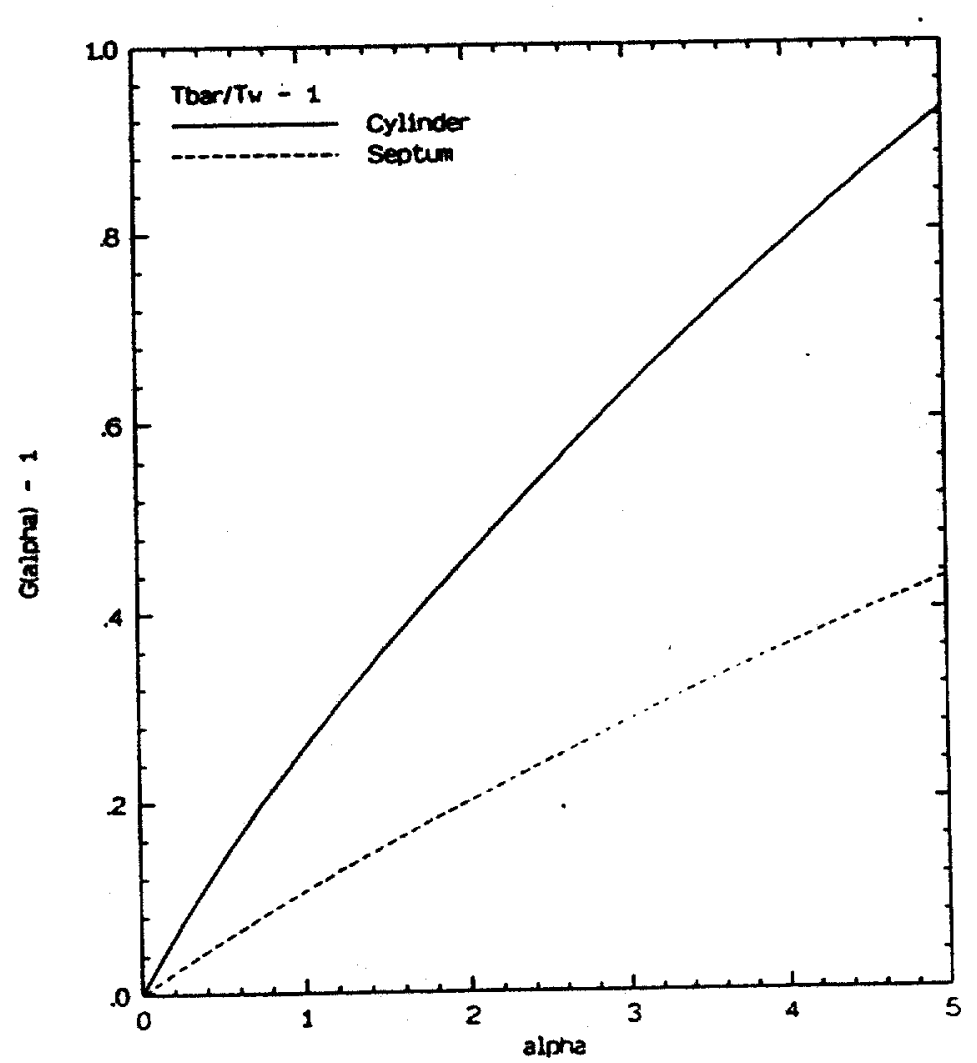

Figure 2: Temperature correction factor $G$ versus deposition parameter $\alpha$, for the laser geometries. Note that $G(\alpha)-1$ unity is plotted. 


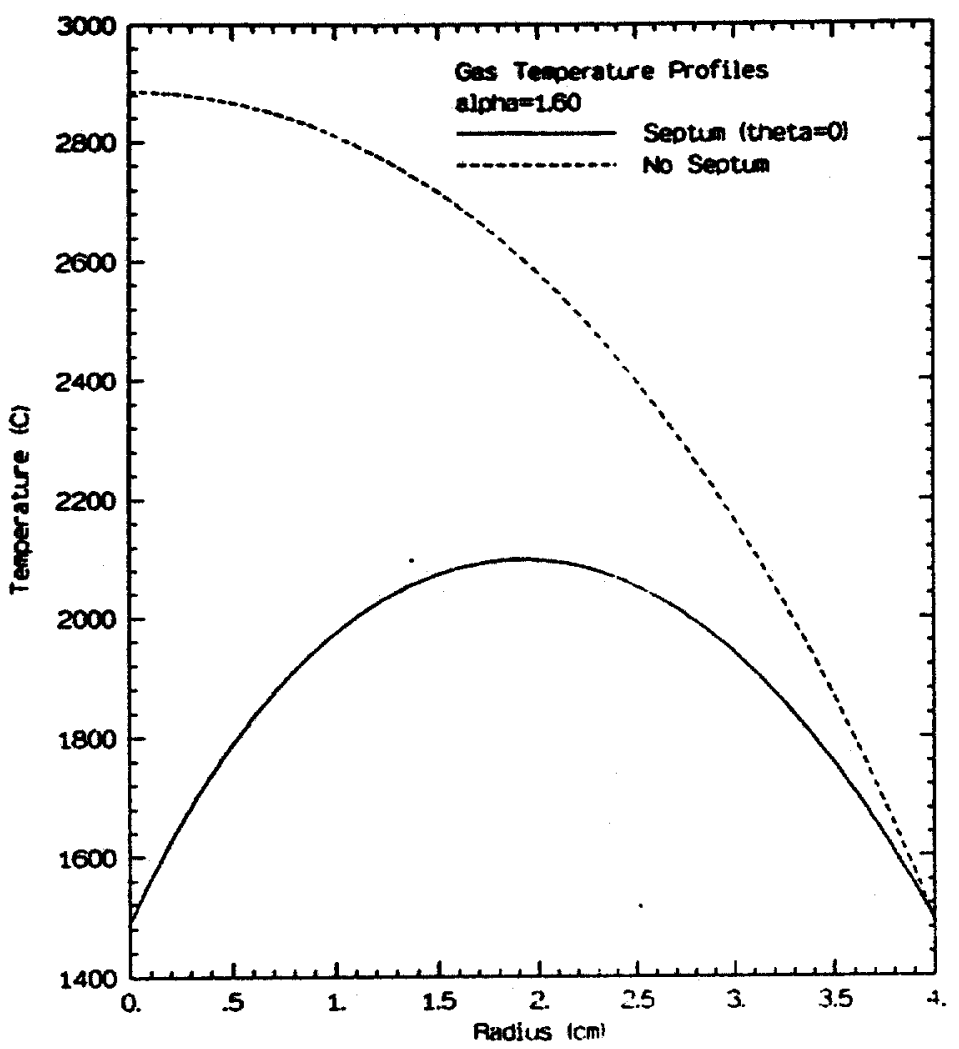

Figure 3: Typical gas temperature profile for the laser geometries. In the septum case, the profile is in the perpendicular midplane. 


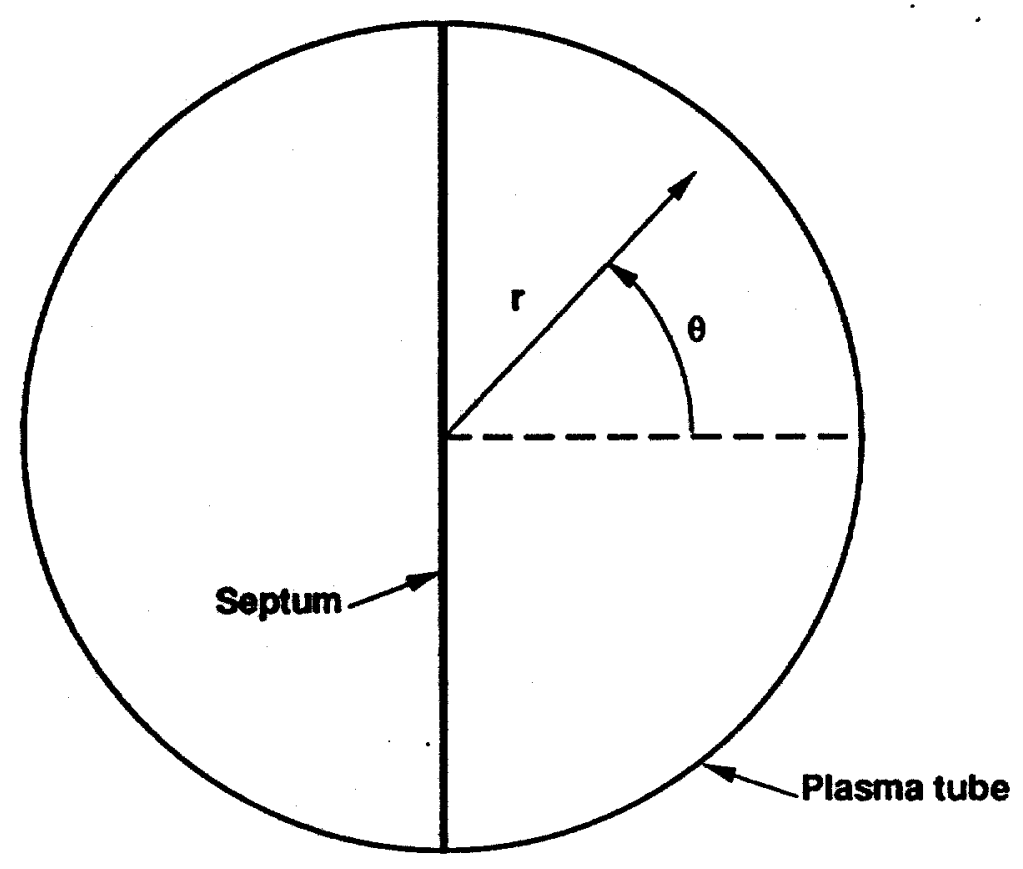
1.1.2.0024.0ess pub

Figure 4: Schematic of septum geometry. 


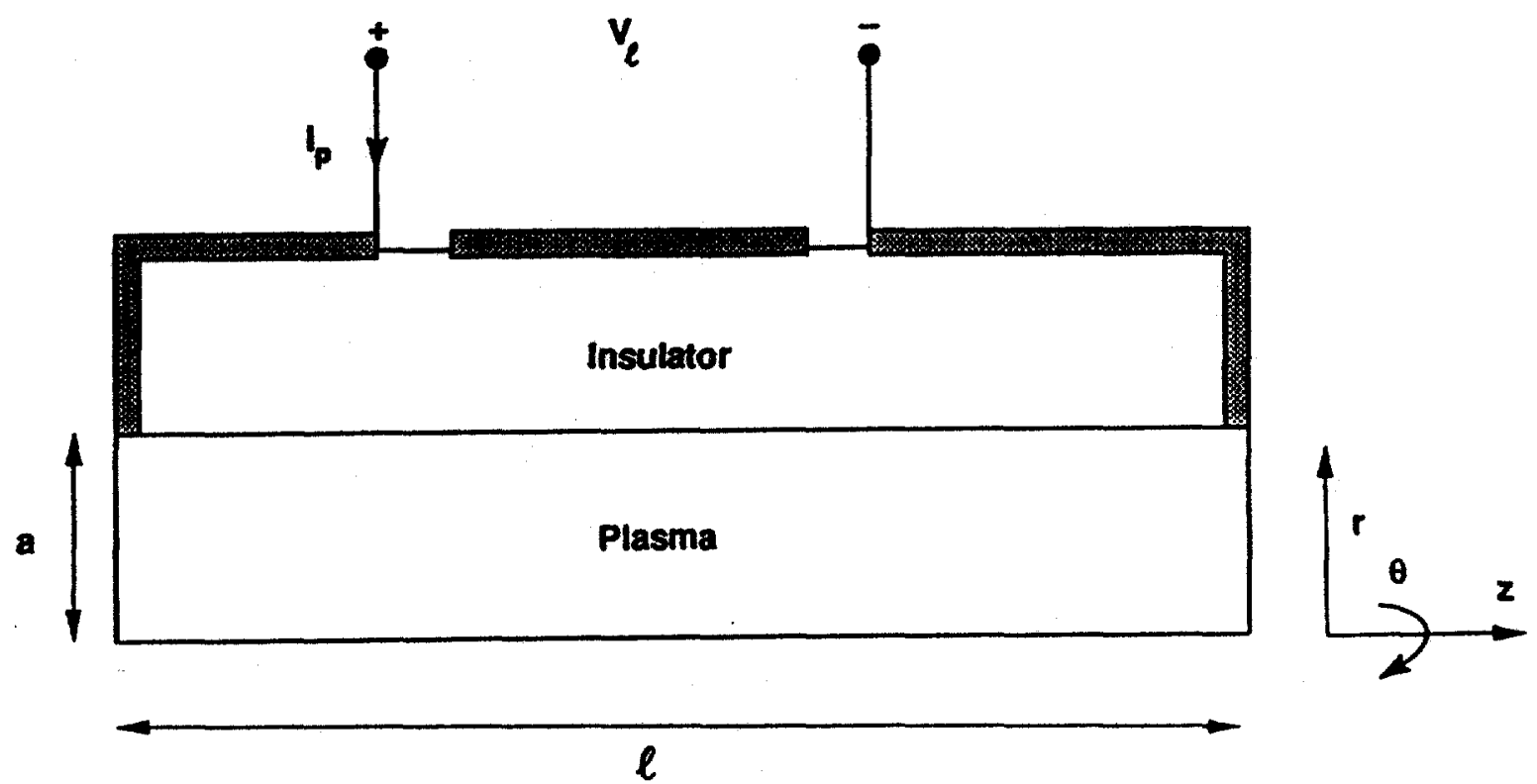

Figure 5: Schematic of laser assembly. 

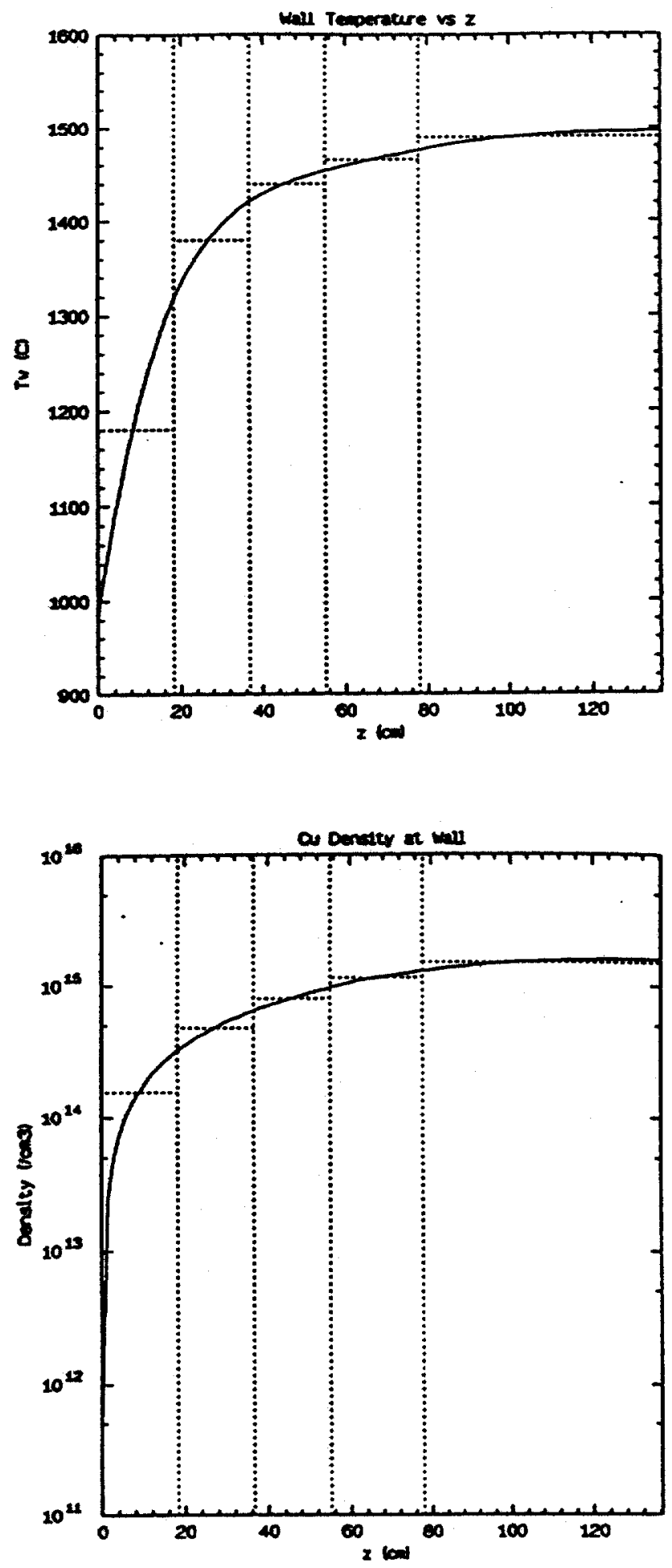

Figure 6: Typical profiles of wall temperature and edge copper density for a septum laser. 

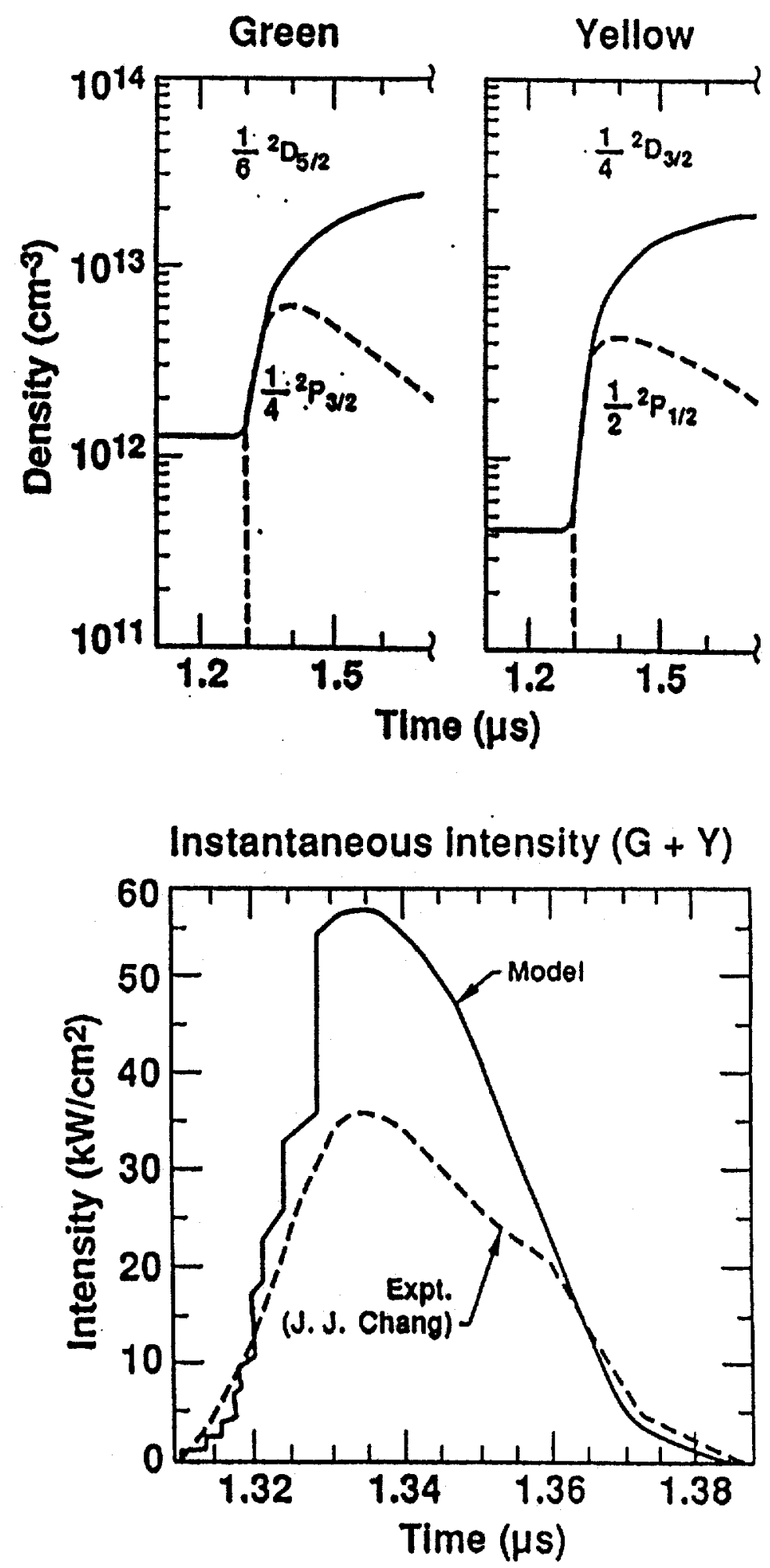

Figure 7: Calculated line-averaged lasing densities and amplified optical signal for a septum laser. 

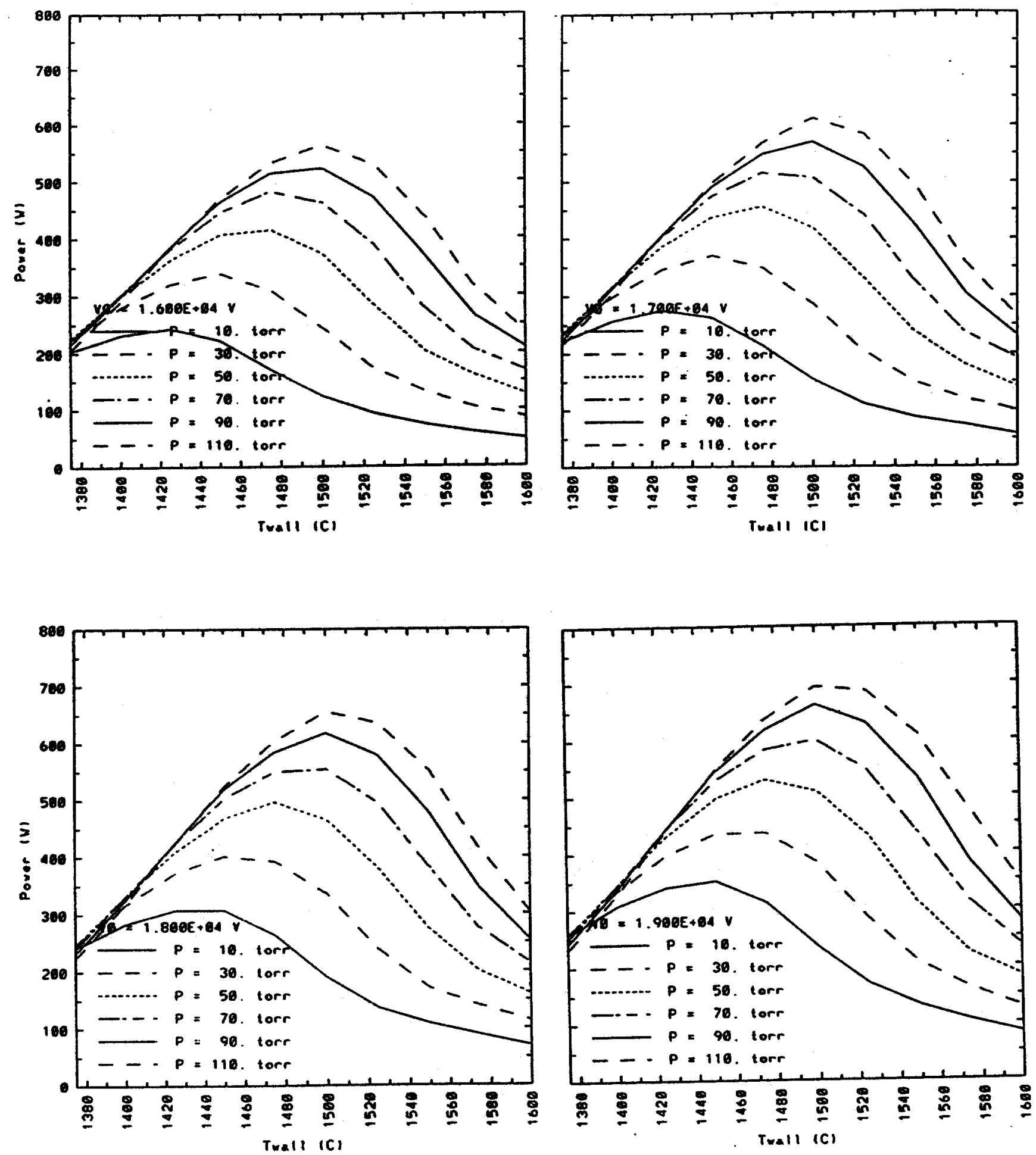

Figure 8: Calculated power versus wall temperature at selected voltages and pressures, for a septum laser. 

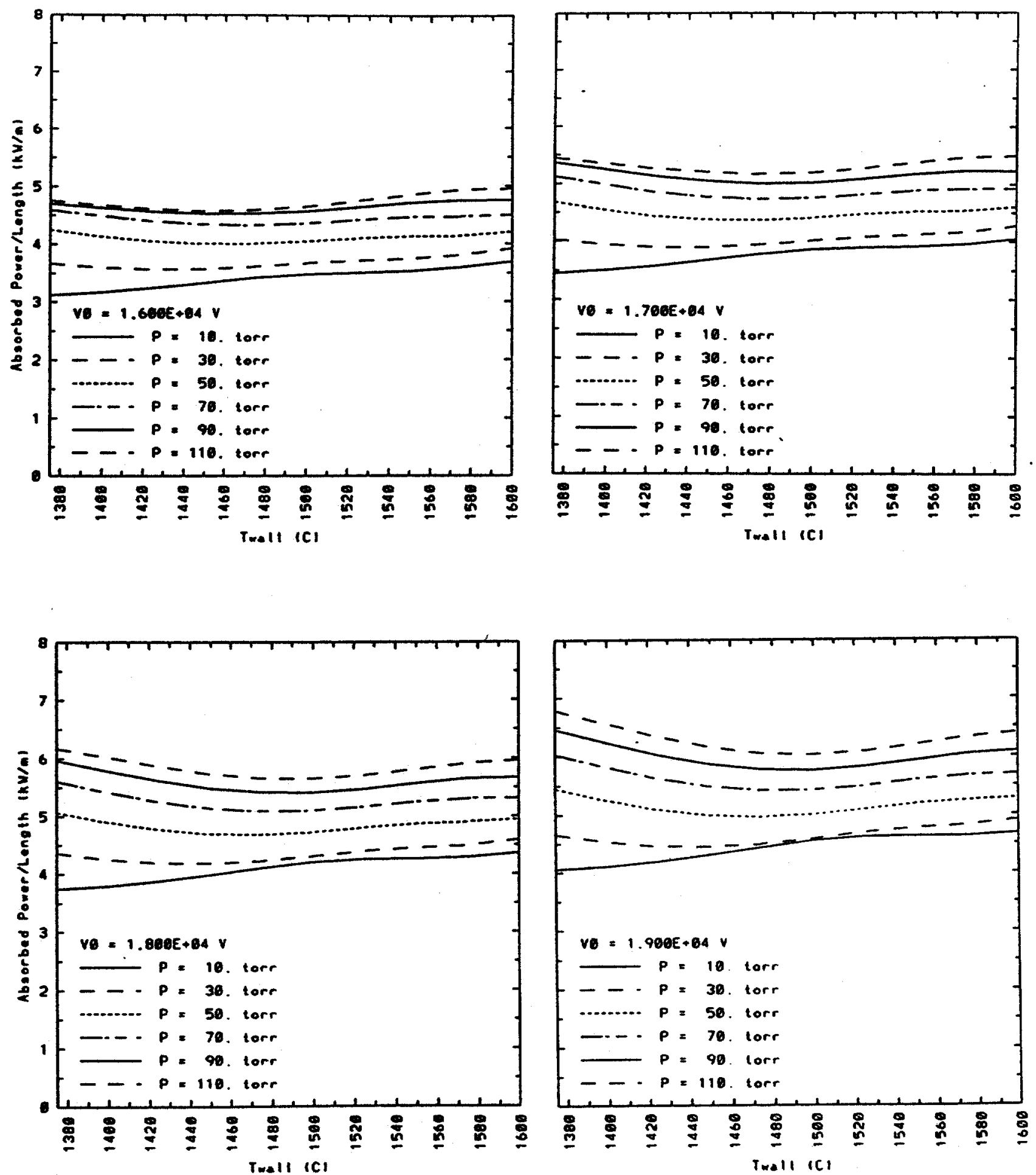

Figure 9: Absorbed power versus wall temperature for a septum laser. 

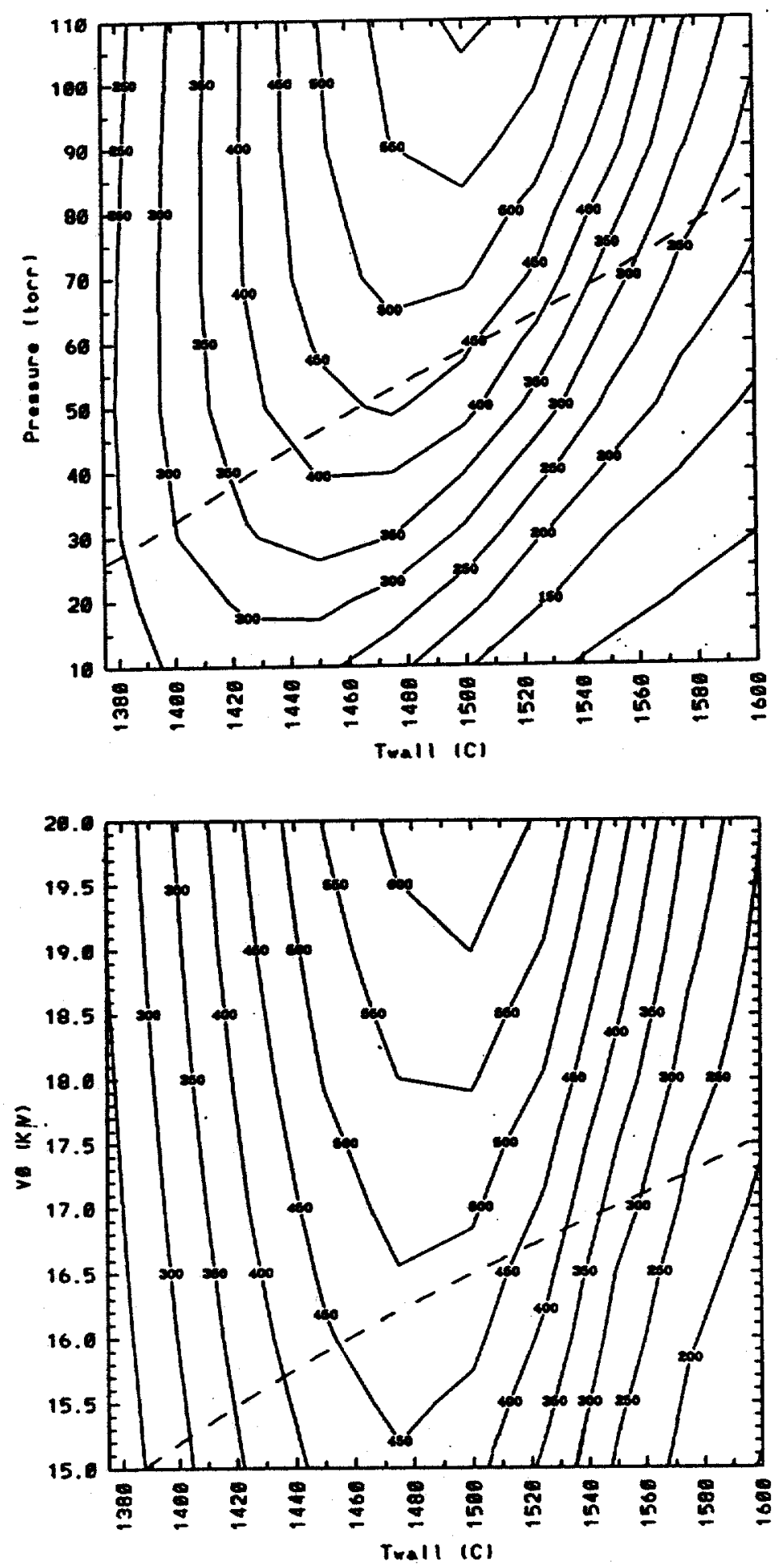

Figure 10: Calculated septum laser power contours. Top: temperature-pressure plane $\left(V_{0}=17 \mathrm{kV}\right)$; bottom: temperature-voltage plane $(p=70$ torr $)$. The dotted lines show points available to experiment, with the standard thermal package. 


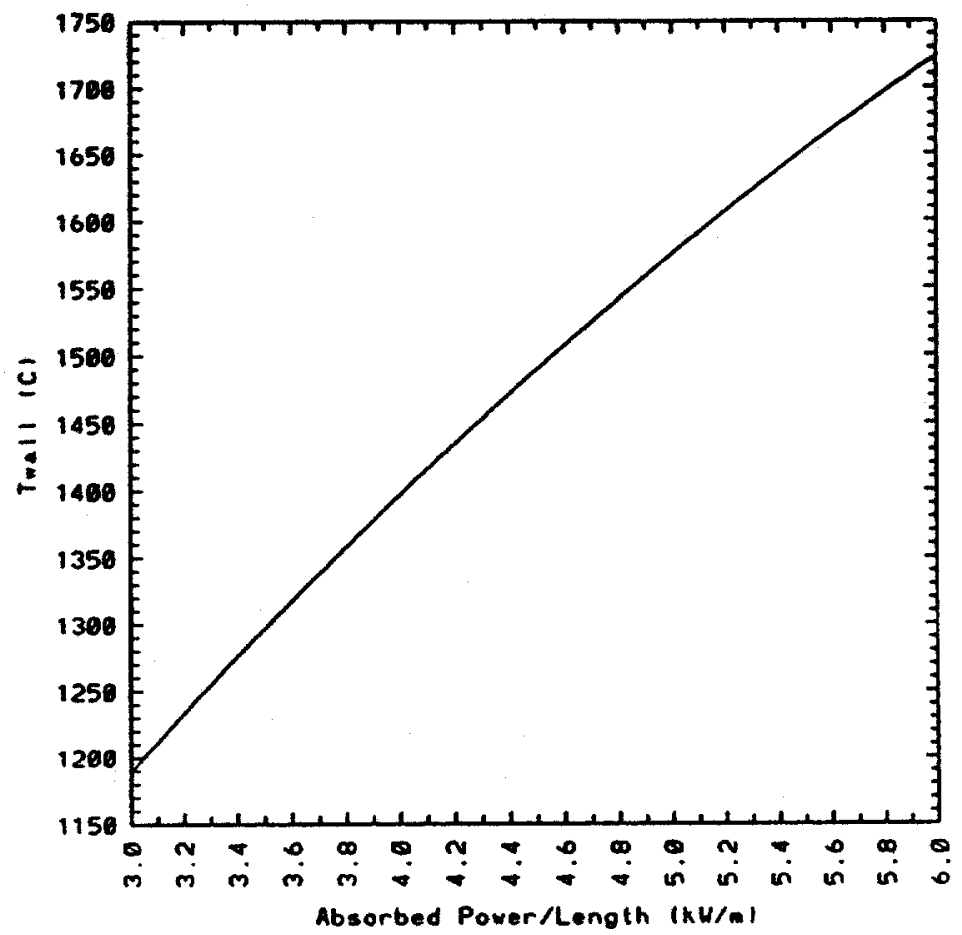

Figure 11: Thermal characteristics of septum laser insulation package midway along the tube. 

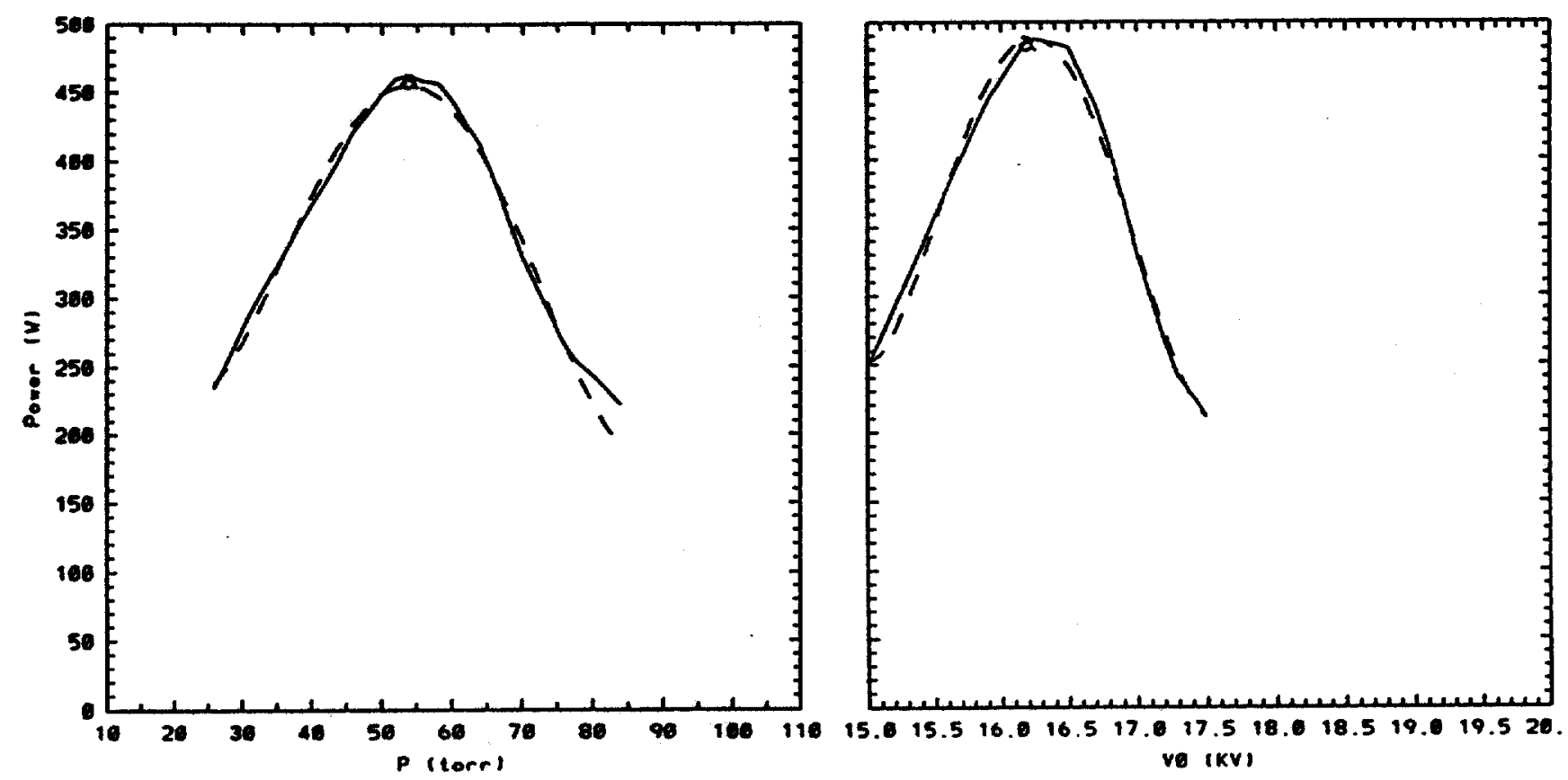

Figure 12: Laser power along the trajectories indicated in Fig. 10. The dashed line indicates a least-squares fit. 

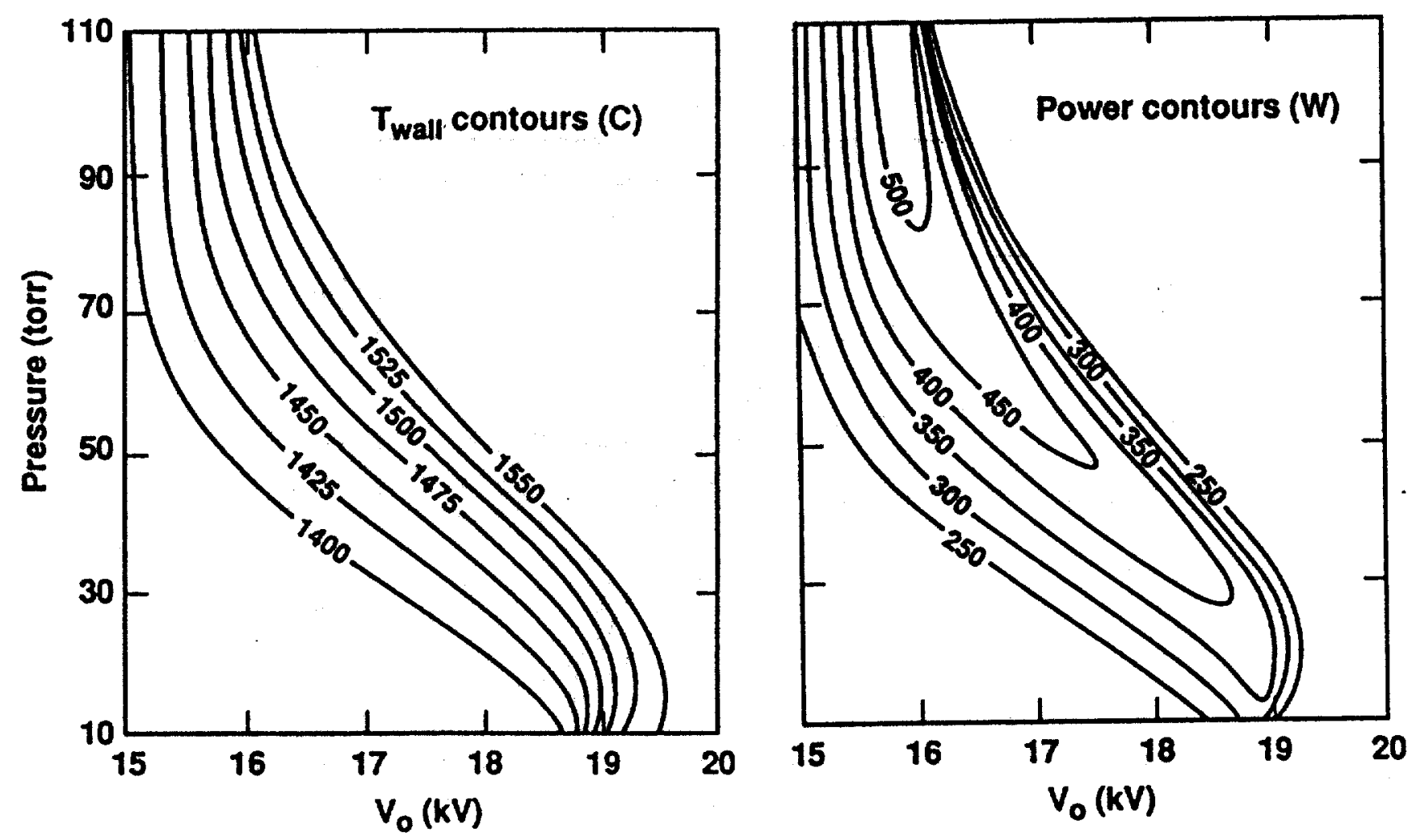

Figure 13: Calculated contours of wall temperature and laser power, on the voltage-pressure plane, for a septum laser. 

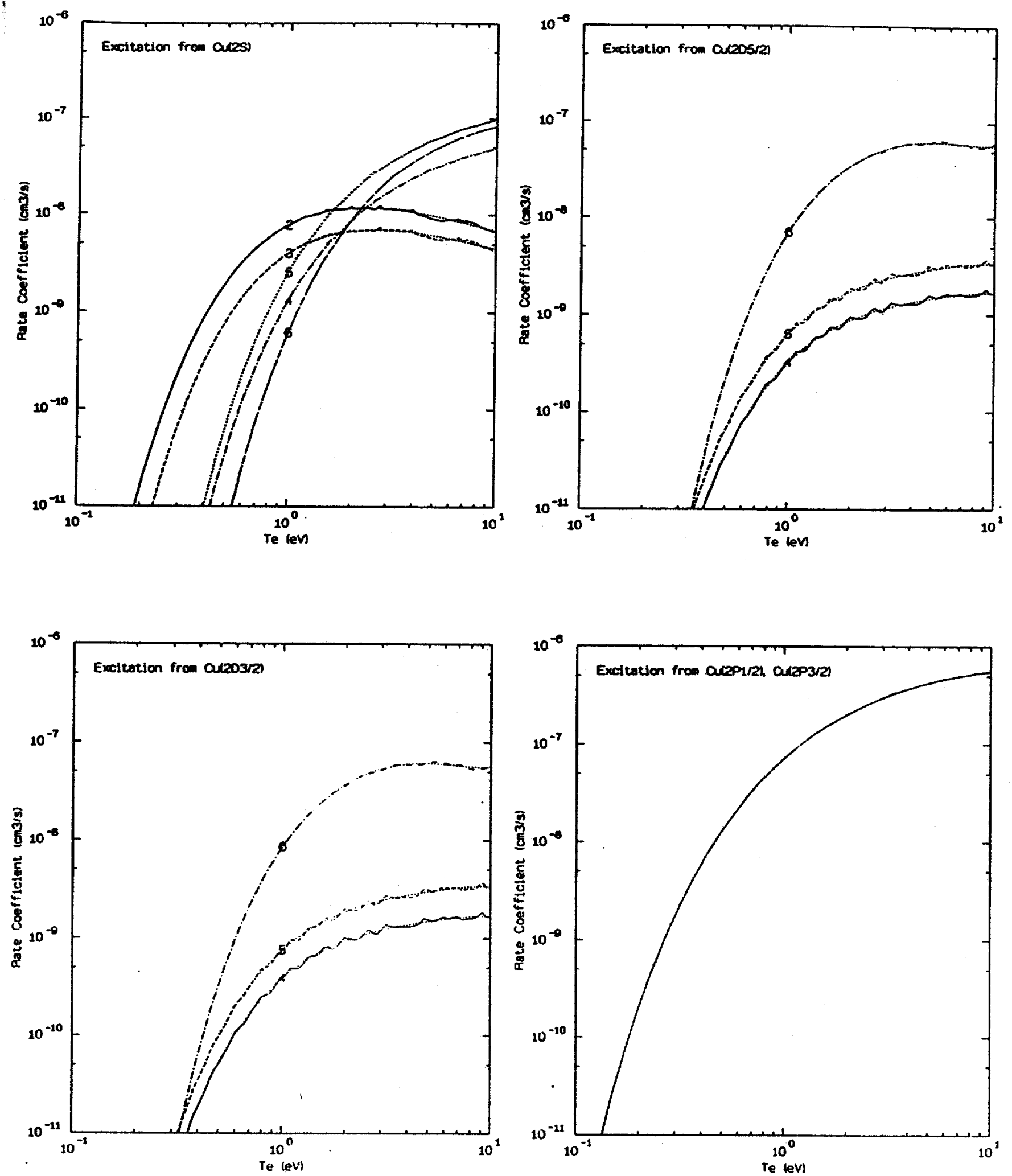

Figure 15: Electron excitation rates from the states of the copper model. The numbers label the final states as in Table 1. The smooth lines lines superimposed on each curve indicate the least squares fit. 

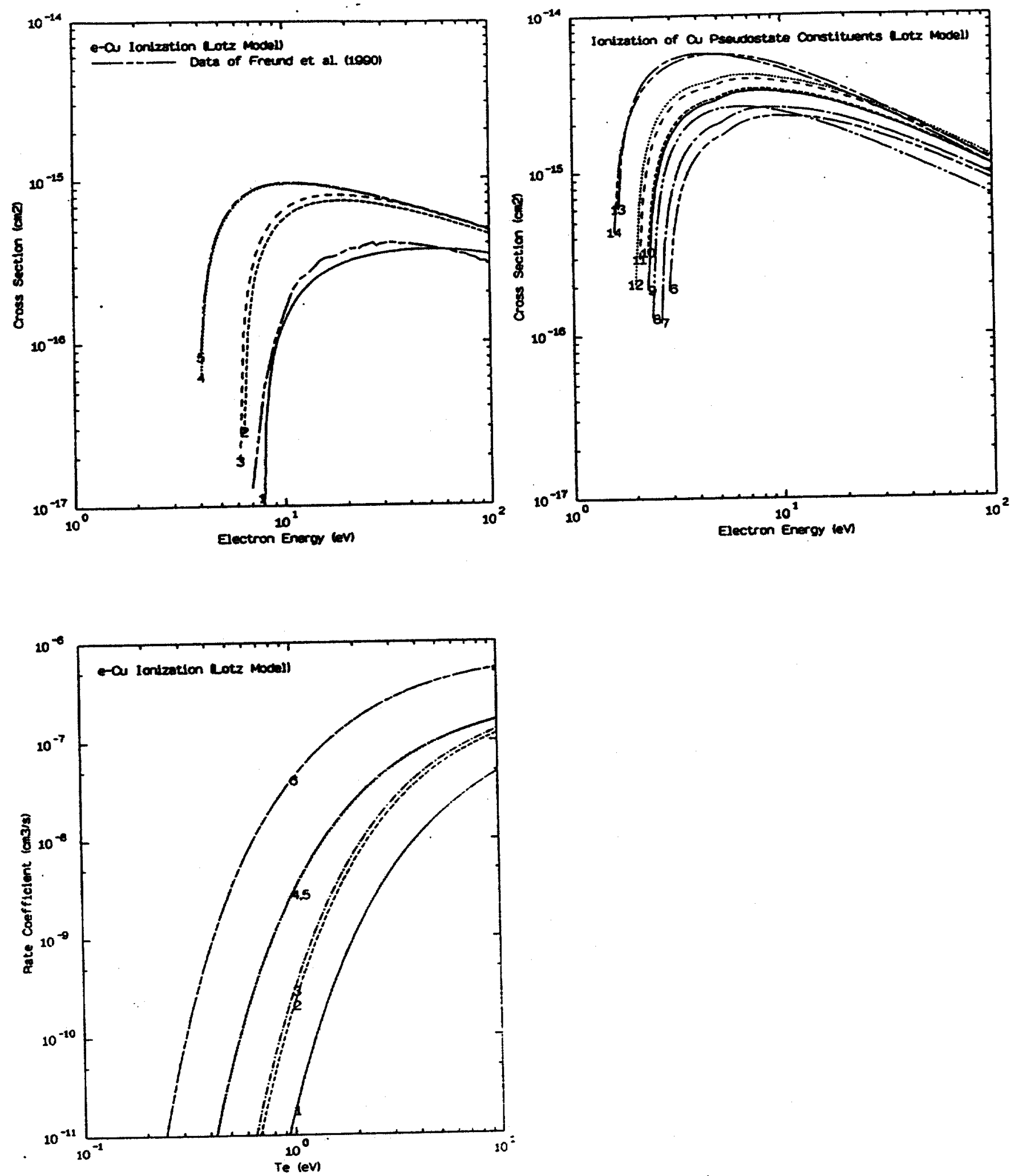

Figure 16: Electron-impact ionization cross sections and rates for the copper model. The numbers label the final states as in Table 1. Also shown are ground state ionization data of Freund et al.. 

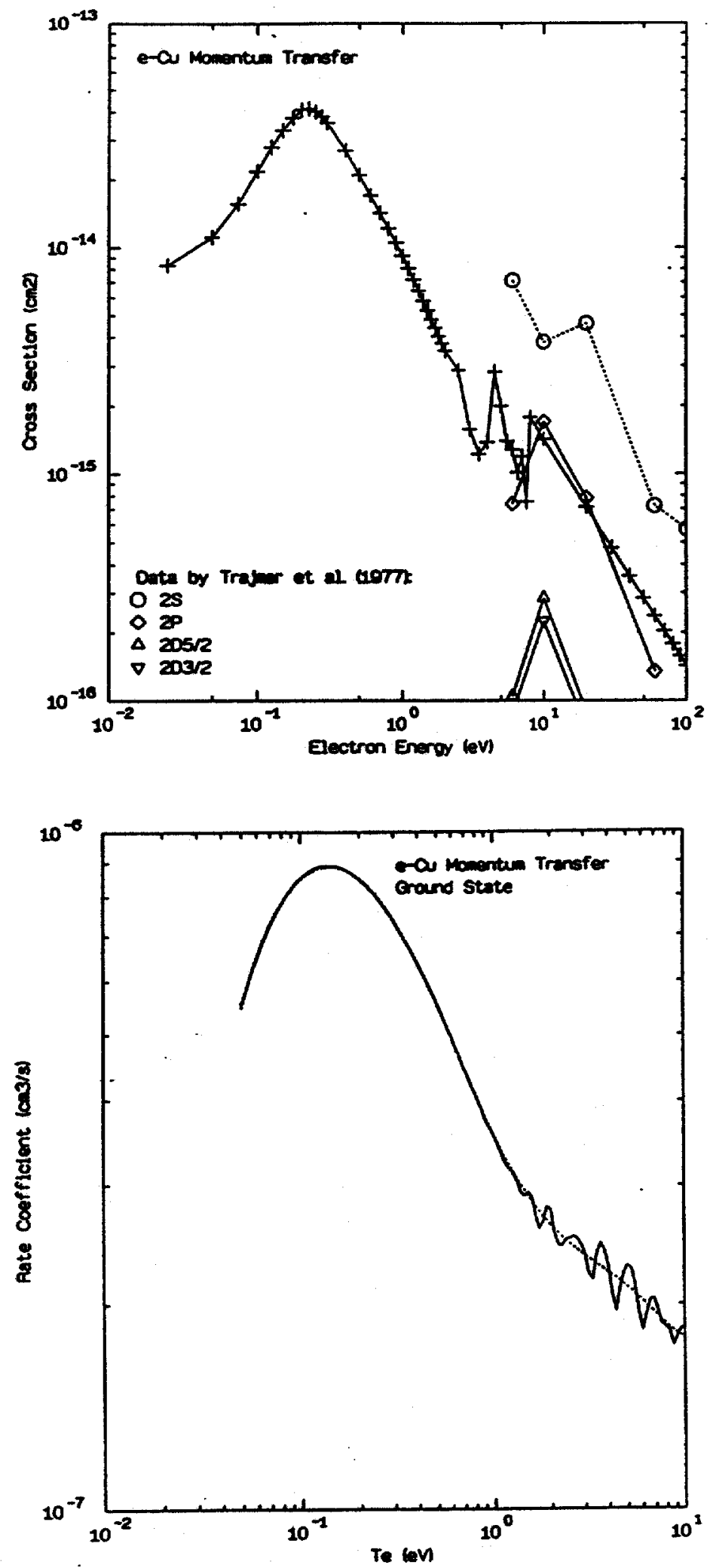

Figure 17: Electron-impact momentum transfer cross sections and rates for the copper model. Also shown are data by Trajmar. The smooth lines line superimposed on the rate indicate the least squares fit. 


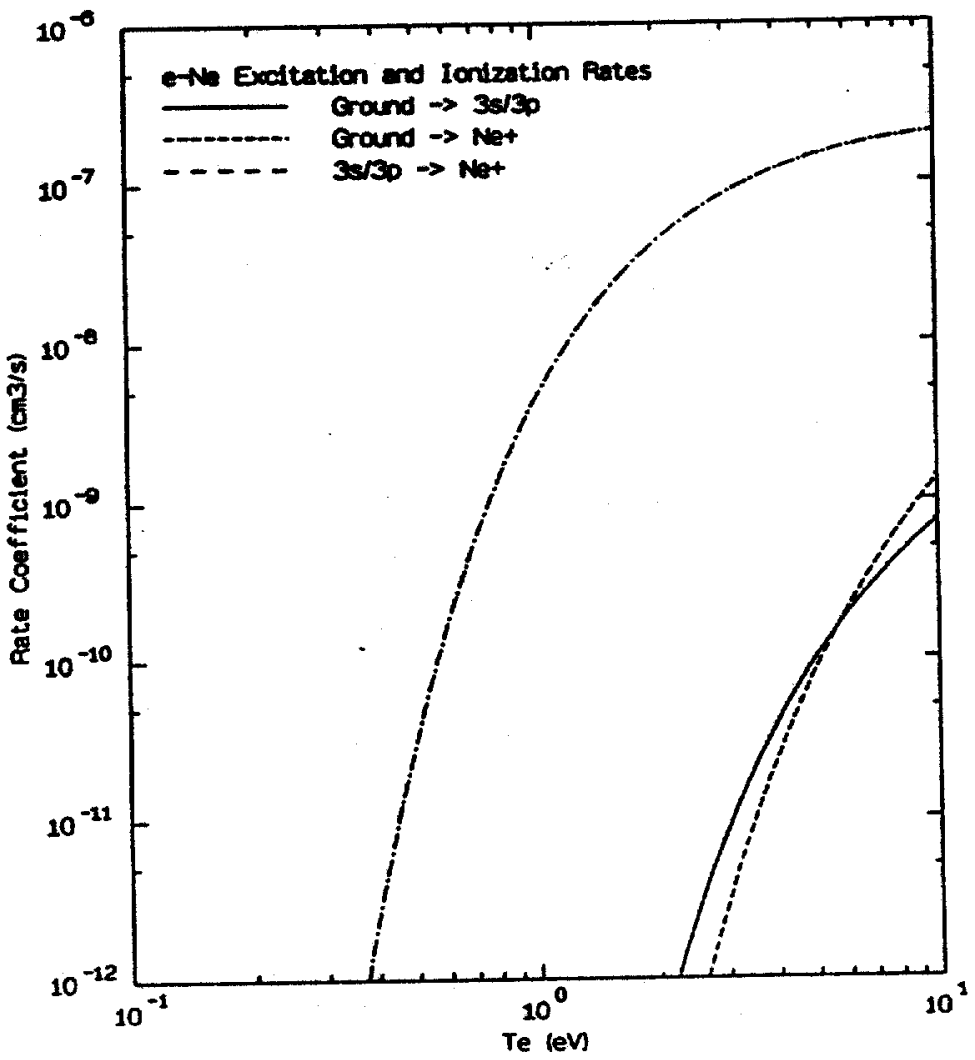

Figure 18: Neon excitation and ionization rates. 

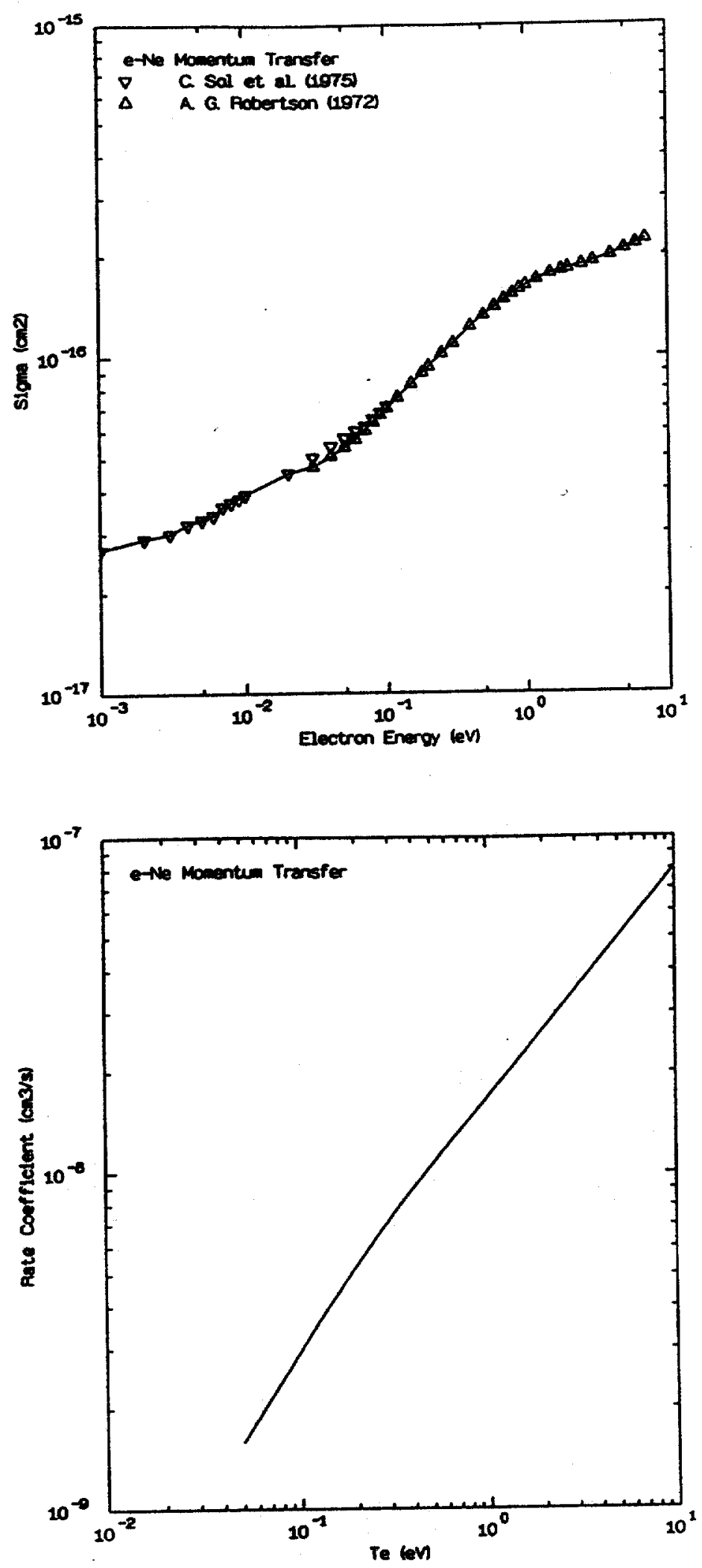

Figure 19: Neon momentum transfer cross section and rate. 

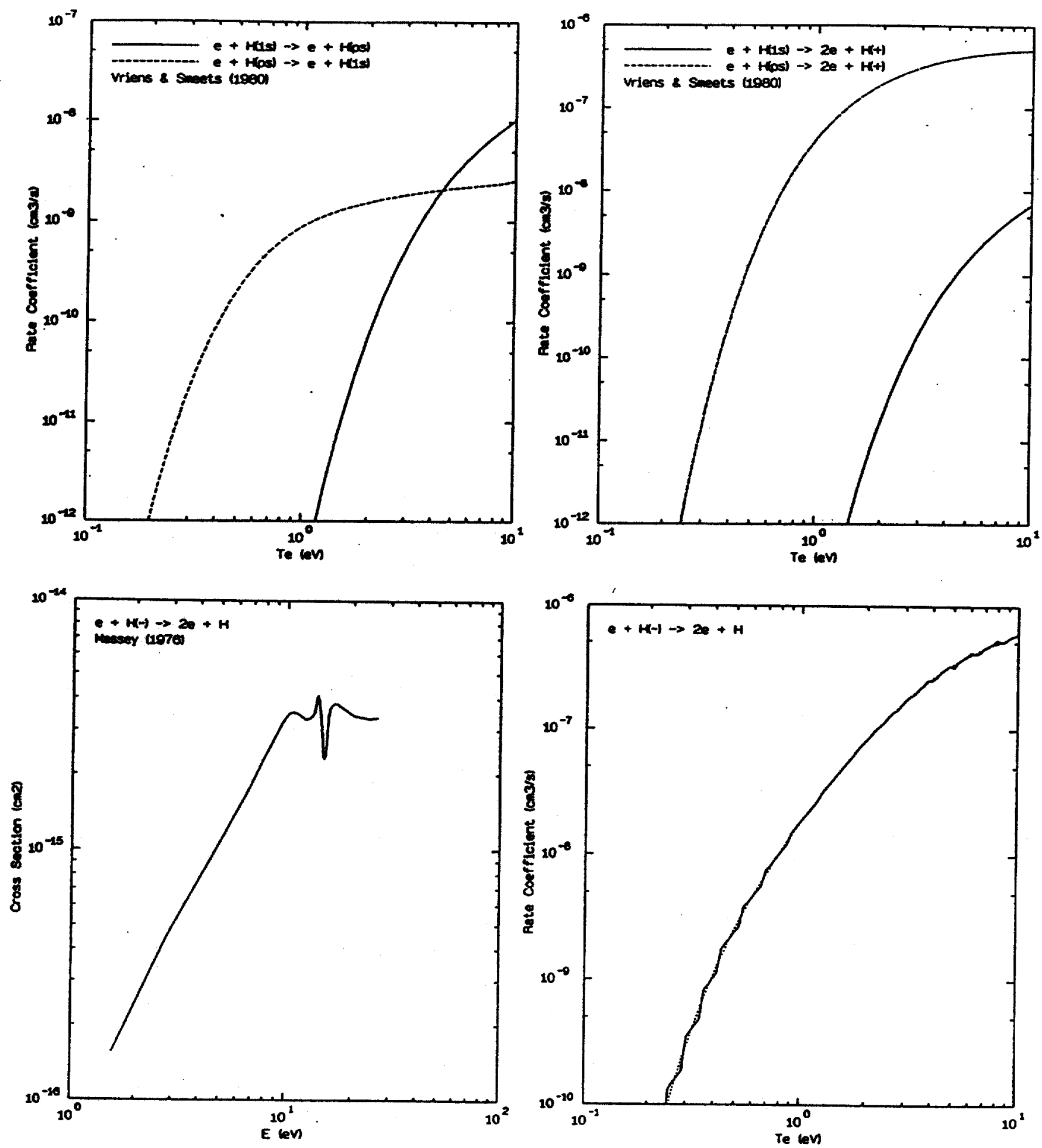

Figure 20: Hydrogen excitation and deexcitation rates; hydrogen ionization rates; negative ion detachment cross section and rate. The smooth lines line superimposed on the last curve indicates the least squares fit. 

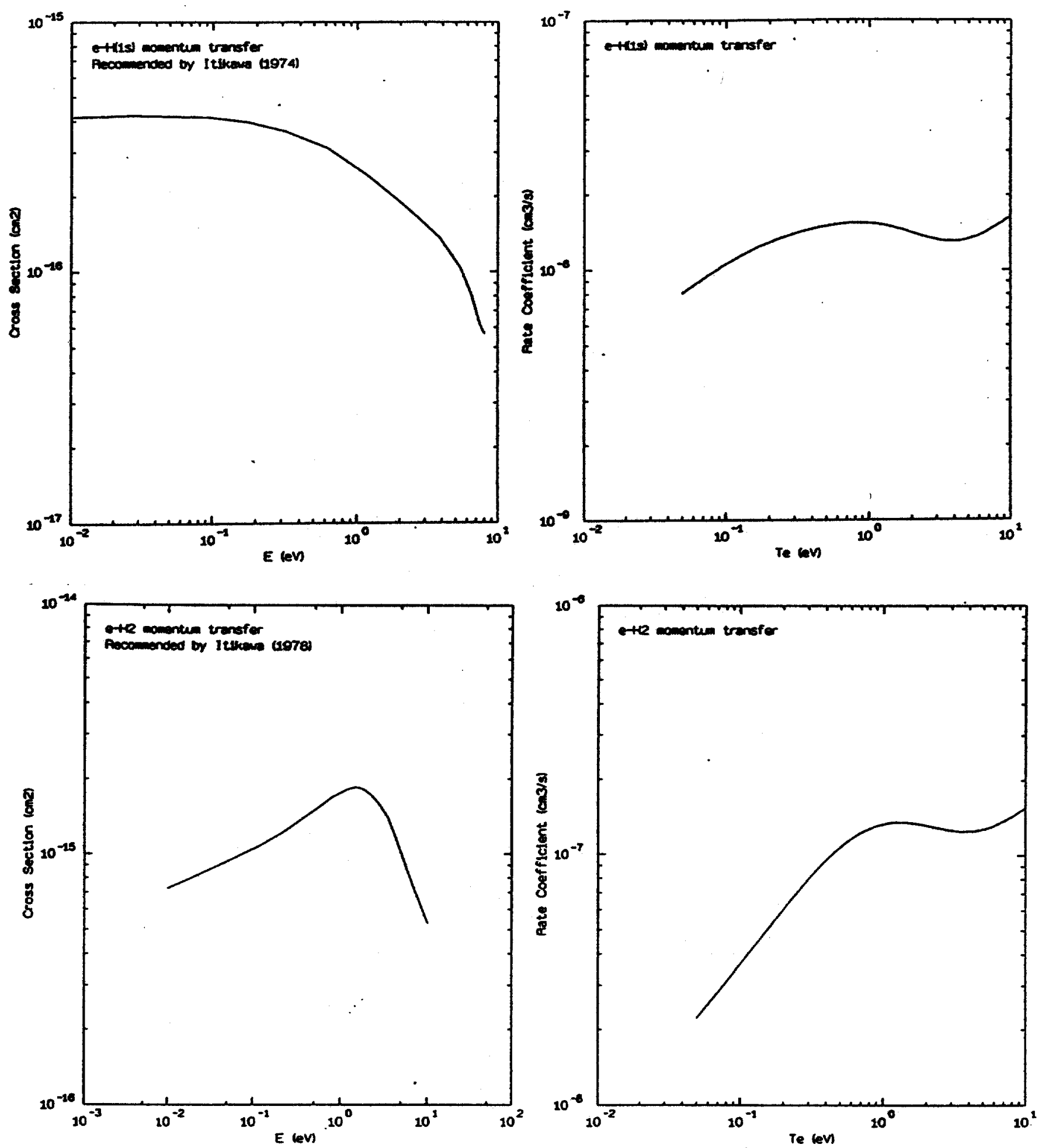

Figure 21: Cross section and rate for momentum transfer in atomic hydrogen and in molecular hydrogen. 


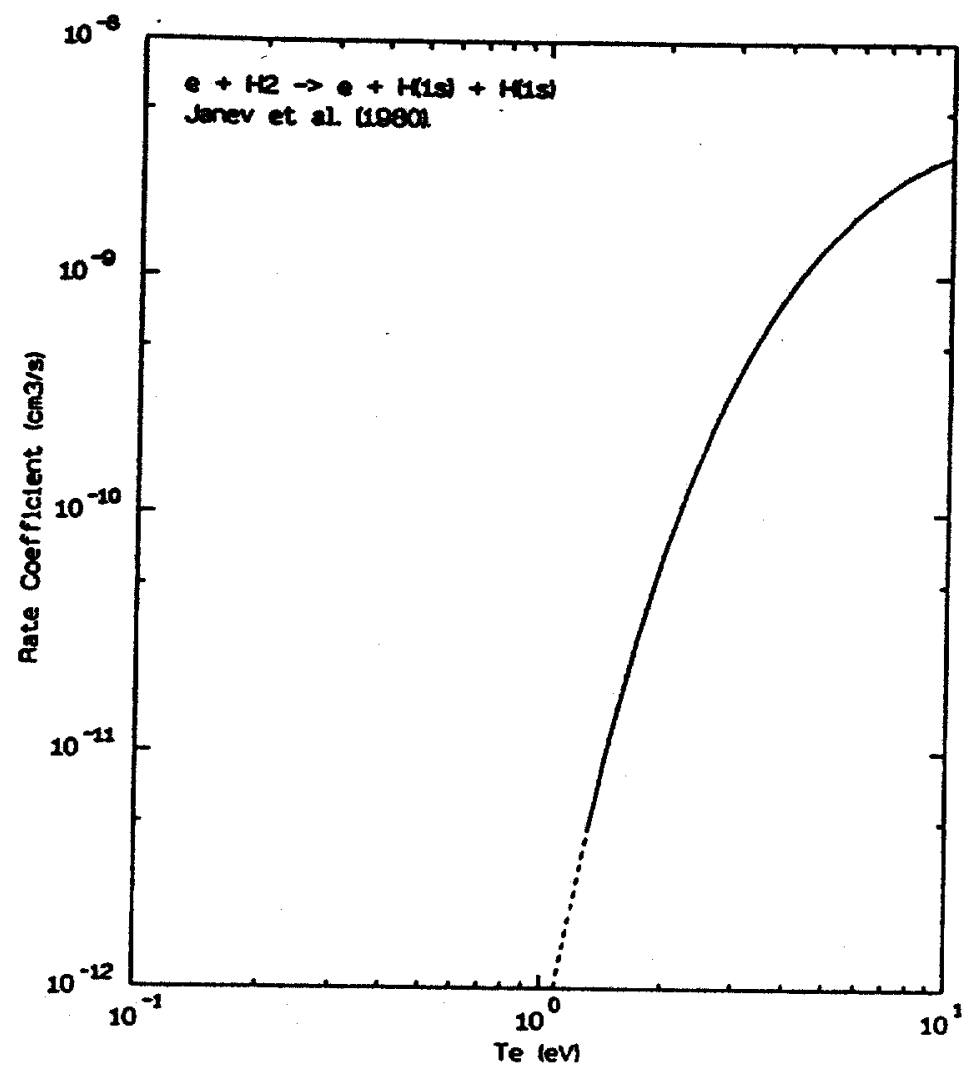

Figure 22: Dissociation rate for molecular hydrogen. 


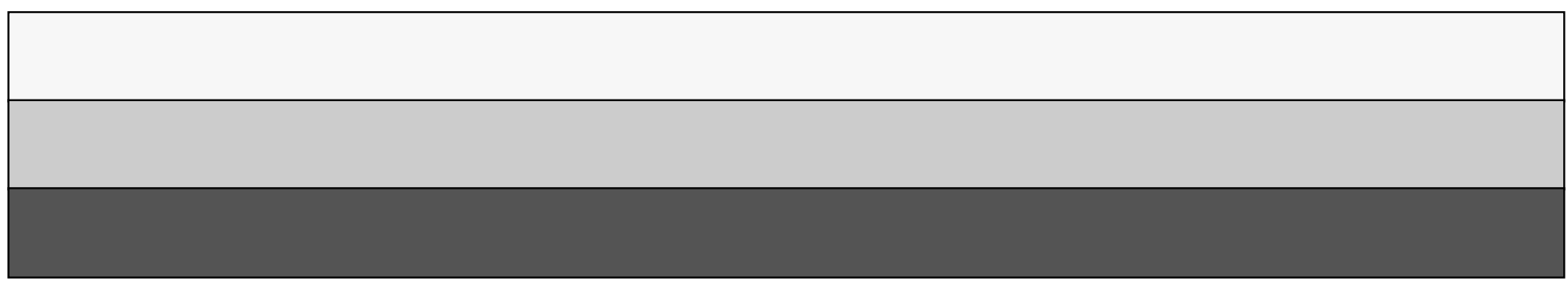

